\title{
THE WEIGHTED POINTWISE ERGODIC THEOREM AND THE INDIVIDUAL ERGODIC THEOREM ALONG SUBSEQUENCES
}

\author{
BY \\ A. BELLOW AND V. LOSERT ${ }^{1}$
}

\begin{abstract}
In this paper on the weighted pointwise ergodic theorem we bring together a substantial amount of previous work by a number of mathematicians and integrate it into a systematic consistent scheme; we also bring some original contributions to the subject which extend its boundaries and suggest further avenues of research. The paper is divided into six sections. The most significant new results are contained in $\S \S 5$ and 6 .
\end{abstract}

1. Introduction. We denote by $\mathbf{N}$ the set of all nonnegative integers and by $\mathbf{Z}$ the set of all integers.

Throughout this paper $(\Omega, \mathcal{A}, \mu)$ is a probability space; for the sake of simplicity we assume that $\Omega=[0,1)(\bmod 1)$ endowed with the $\sigma$-field of Borel sets and Lebesgue measure.

Let $C$ be the group of automorphisms of $(\Omega, \mathcal{A}, \mu) ; T \in C$ if $T: \Omega \rightarrow \Omega$ is a bijection which is bimeasurable and preserves $\mu$. In what follows we shall single out various classes of automorphisms such as the ergodic automorphisms, the automorphisms with discrete spectrum, the automorphisms with Lebesgue spectrum and the $K$ automorphisms (see [38]). via

Let $T \in C$ and let $1 \leq p \leq \infty ; T$ induces an operator $U_{T}$ in $L^{p}(\Omega)=L^{p}(\Omega, \mathcal{A}, \mu)$

$$
f \rightarrow f \circ T \text { for } f \in L^{p}(\Omega) \text {. }
$$

For $p=2$, the induced operator $U_{T}$ is a unitary operator; we shall often make use of its spectral resolution.

Now let $T$ be a continuous linear operator on $L^{p}(\Omega)$ for some $1 \leq p<\infty$. Let $\mathbf{a}=\left(a_{n}\right)$ be a sequence of complex numbers.

Received by the editors November 10, 1983.

1980 Mathematics Subject Classification. Primary 28D05, 60F 15, 60G10, 43A60.

Key words and phrases. Automorphism, ergodic, discrete spectrum, countable Lebesgue spectrum, $K$-automorphism, "good universal weight", positive definite function, affinity of two probability measures (=Hellinger integral), correlation of a sequence, spectral measure corresponding to a sequence, Besicovitch class $B(p)$, almost periodic functions (in the sense of Bohr, Weyl, Eberlein), Bochner-Fejér polynomial, strictly $L$-stable dynamical system, "uniform sequence", Bohr compactification, sequence that satisfies a "uniform order conditin on $J$ ", "saturating sequence", Weak Maximal Inequality, "bad universal sequence", "block sequence", lacunary sequence, "good universal sequence" of density zero.

${ }^{1}$ Research supported by the National Science Foundation (U.S.A.). 
DeFinition 1.1. We say that $\mathbf{a}=\left(a_{n}\right)$ is a good weight in $L^{p}$ for $T$ (relative to the Weighted Pointwise Ergodic Theorem) if, for every $f \in L^{p}(\Omega)$,

$$
\lim _{n} \frac{1}{n} \sum_{j=0}^{n-1} a_{j} T^{j} f(\omega) \quad \text { exists } \mu \text {-a.e. }
$$

By a slight abuse of language. in the case when $S \in \mathbf{C}$, we say that $\mathbf{a}=\left(a_{n}\right)$ is a good weight for $S$ in $L^{1}$, or simply that a is a good weight for $S$ if a is a good weight in $L^{1}$ for the operator $U_{S}$ induced by $S$.

Definition 1.2. We say that the Maximal Inequality holds in $L^{p}$ for $(T, \mathbf{a})$ if, setting

$$
M f(\omega)=\sup _{n \geq 1}\left|\frac{1}{n} \sum_{j=0}^{n-1} a_{j} T^{j} f(\omega)\right| .
$$

we have

$$
\lambda \mu(\{M f>\lambda\}) \leq(\text { constant })\|f\|_{p}
$$

for all $f \in L^{p}(\Omega)$ and $\lambda>0$.

We recall that $T: L^{1}(\Omega) \rightarrow L^{1}(\Omega)$ is called a Dunford-Schwartz operator if $T$ is a contraction both for the $L^{1}$ and the $L^{\infty}$ norm; then $T \mid L^{p}(\Omega)$ is also a contraction for the $L^{p}$ norm, $1<p<\infty$. We have (see [17]; see also [28])

Proposition 1.3. Let $T$ be a Dunford-Schwartz operator, let $\mathbf{a}=\left(a_{n}\right)$ be a bounded sequence of complex numbers and let $1 \leq p<\infty$. Then:

(1) the Maximal Inequality holds in $L^{p}$ for $(T, \mathbf{a})$;

(2) the set $E$ of all $f \in L^{p}(\Omega)$ for which

$$
\lim _{n \rightarrow \infty} \frac{1}{n} \sum_{j=0}^{n-1} a_{j} T^{j} f(\omega) \quad \text { exists } \mu \text {-a.e. }
$$

is a closed vector space in $L^{p}(\Omega)$ and the linear operator $P: E \rightarrow L^{p}(\Omega)$ defined by

$$
P f(\omega)=\lim _{n} \frac{1}{n} \sum_{j=0}^{n-1} a_{j} T^{j} f(\omega)
$$

is bounded.

REMARK. Statement (2) in the previous proposition is nothing else than $\mathrm{Ba}$ nach's principle (see $[\mathbf{1 7}$ or $\mathbf{2 6}]$ ) formulated in our context.

THEOREM $1.4[\mathbf{2}]$. Let $\mathbf{a}=\left(a_{n}\right)$ be a bounded complex sequence. The following assertions are equivalent:

(i) The sequence a is a good weight in $L^{1}$ for every (operator induced by some) ergodic $S \in C$.

(ii) The sequence a is a good weight in $L^{1}$ for every (operator induced by some) $S \in C$.

(iii) The sequence a is a good weight in $L^{1}$ for every Dunford-Schwartz operator.

REMARK. This striking theorem is essentially due to Baxter and Olsen, that is, the equivalence (ii) $\Leftrightarrow$ (iii). To show that (i) $\Rightarrow$ (ii), use "decomposition into ergodic 
parts" (see [35], or see [37] for a concise and elegant treatment of what is needed here).

DEFINITION 1.5. A bounded complex sequence $\mathbf{a}=\left(a_{n}\right)$ is said to be a "good universal weight" if a is a good weight in $L^{1}$ for every Dunford-Schwartz operator (equivalently, by Theorem 1.4, for every operator induced by some $S \in C$ ergodic).

We shall also be interested in the Individual Ergodic Theorem along subsequences. In what follows $\mathbf{n}=\left(n_{k}\right)$ will always denote a strictly increasing sequence of nonnegative integers. If $\mathbf{m}$ is a subsequence of $\mathbf{n}$ we write $\mathbf{m} \subset \mathbf{n}$.

By analogy with "weights" we may define the notion of "good subsequence for $T$ " and "good universal subsequence". For instance,

DEFINITION 1.6. We say that $\mathbf{n}=\left(n_{k}\right)$ is a good subsequence for $T$ in $L^{p}$ (relative to the Pointwise Ergodic Theorem) if for every $f \in L^{p}(\Omega)$

$$
\lim _{K \rightarrow \infty} \frac{1}{K} \sum_{k=0}^{K-1} T^{n_{k}} f(\omega) \text { exists } \mu \text {-a.e. }
$$

If the subsequence $\mathbf{n}$ has nonzero density, then the Pointwise Ergodic Theorem along $\mathbf{n}$ may be reduced to a Weighted Pointwise Ergodic Theorem. In fact,

PROPOSITION 1.7. Suppose the subsequence $\mathbf{n}=\left(n_{k}\right)$ has density $d>0$. Define the weight $\mathbf{a}=\left(a_{n}\right)$ by $a_{n}=1$ if $n=n_{k}$ for some $k$, and $a_{n}=0$ otherwise. Then for any complex sequence $\left(u_{n}\right)$ we have

$$
\text { d. } \lim _{K \rightarrow \infty} \frac{1}{K} \sum_{k=0}^{K-1} u_{n_{k}}=\lim _{n \rightarrow \infty} \frac{1}{n} \sum_{j=0}^{n-1} a_{j} u_{j},
$$

that is, the existence of either limit implies the existence of the other, with the equality above.

For this reason, we shall deal mostly with the Weighted Pointwise Ergodic Theorem.

Since we shall repeatedly use the "subsequence argument" in what follows, we recall this here.

DEFINITION 1.8. An increasing sequence $\left(N_{k}\right)$ of positive integers is said to be dense in $\mathbf{N}$ if

$$
\lim _{k \rightarrow \infty} \frac{N_{k+1}}{N_{k}}=1 .
$$

We shall need the following elementary result.

PROPOSITION 1.9. Let $\left(u_{n}\right)$ and $\left(v_{n}\right)$ be two sequences of complex numbers. We assume that

(1) the sequence $\left(u_{n}\right)$ is bounded;

(2) the sequence $\left(\frac{1}{n} \sum_{k=0}^{n-1}\left|v_{k}\right|^{2}\right)$ is bounded;

(3) there exists a sequence $\left(N_{k}\right)$ of positive integers, dense in $\mathbf{N}$, such that

$$
\lim _{k \rightarrow \infty} \frac{1}{N_{k}} \sum_{j=0}^{N_{k-1}} u_{j} v_{j} \quad \text { exists and }=A \text {. }
$$

Then

$$
\lim _{n \rightarrow \infty} \frac{1}{n} \sum_{j=0}^{n-1} u_{j} v_{j} \quad \text { exists and }=A
$$


PROOF. If $N_{k}<n \leq N_{k+1}$, then

$$
\frac{1}{n} \sum_{j=0}^{n-1} u_{j} v_{j}=\frac{N_{k}}{n} \frac{1}{N_{k}} \sum_{j=0}^{N_{k}-1} u_{j} v_{j}+\frac{1}{n} \sum_{j=N_{k}}^{n-1} u_{j} v_{j} .
$$

The first term on the right clearly tends to $A$. As for the second, we have

$$
\begin{aligned}
\left|\frac{1}{n} \sum_{j=N_{k}}^{n-1} u_{j} v_{j}\right|^{2} & \leq\left(\frac{1}{n} \sum_{j=N_{k}}^{n-1}\left|u_{j}\right|^{2}\right)\left(\frac{1}{n} \sum_{j=N_{k}}^{n-1}\left|v_{j}\right|^{2}\right) \leq C_{1}\left(\frac{n-N_{k}}{n}\right) \cdot C_{2} \\
& \leq C_{1} C_{2}\left(\frac{N_{k+1}-N_{k}}{N_{k}}\right)=C_{1} C_{2}\left(\frac{N_{k+1}}{N_{k}}-1\right)
\end{aligned}
$$

and the last term tends to zero.

2. Preliminaries on sequences having a correlation. In what follows we denote by $\mathbf{C}$ the set of all complex numbers and by $\mathbf{C}_{1}$ the unit circle

$$
\mathbf{C}_{1}=\{z \in \mathbf{C}|| z \mid=1\} \text {. }
$$

Usually a sequence of complex numbers is denoted by $\mathbf{a}=\left(a_{k}\right)$; when we want to emphasize that we are dealing with a function on $\mathbf{N}$, we write $f=(f(k))$.

Let $\mathbf{a}=\left(a_{k}\right)$ be a complex sequence. For $1 \leq p<\infty$ define $\|\mathbf{a}\|_{p}$ by

$$
\|\mathbf{a}\|_{p}^{p}=\underset{n}{\limsup } \frac{1}{n} \sum_{k=0}^{n-1}\left|a_{k}\right|^{p}
$$

and let $l(p)=\left\{\mathbf{a} \mid\|\mathbf{a}\|_{p}<\infty\right\} ;$ then $l(p)$ is a vector space and $\|\cdot\|_{p}$ is a seminorm on it. We also define $l(\infty)$ as the space of all bounded complex sequences and $\|\mathbf{a}\|_{\infty}=\sup _{k}\left|a_{k}\right|$ for $\mathbf{a} \in l(\infty)$.

We now consider the space $S$ of complex sequences $\mathbf{a}=\left(a_{k}\right)$ such that

$$
\gamma_{\mathbf{a}}(k)=\lim _{n} \frac{1}{n} \sum_{j=0}^{n-1} a_{j+k} \bar{a}_{j}
$$

exists for each integer $k \in \mathbf{N}$. This space was introduced by $\mathbf{N}$. Wiener in $[\mathbf{3 9}$, Chapter IV]. It is clear that $S \subset l(2)$.

REMARK. A sequence $\mathbf{a} \in S$ does not necessarily have a mean. The following is an example:

Let $\mathbf{a}=\left(a_{k}\right)=\left(e^{2 \pi i \log (k+1)}\right)$. It is known that the sequence $(\log (n+1))$ is not uniformly distributed mod 1 and, in fact, it is shown in [30, Example 2.4, pp. 8-9] that the averages

$$
\frac{1}{n} \sum_{k=0}^{n-1} e^{2 \pi i \log (k+1)}
$$

do not have a limit when $n \rightarrow \infty$. On the other hand, it is easily checked that $\gamma_{\mathbf{a}}(k)$ exists for all $k \in \mathbf{N}$. In fact, for fixed $k$,

$$
\begin{aligned}
\gamma_{\mathbf{a}}(k) & =\lim _{n} \frac{1}{n} \sum_{j=0}^{n-1} a_{j+k} \bar{a}_{j}=\lim _{n} \frac{1}{n} \sum_{j=0}^{n-1} e^{2 \pi i \log ((j+k+1) /(j+1))} \\
& =\lim _{n} \frac{1}{n} \sum_{j=0}^{n-1} e^{2 \pi i \log (1+k /(j+1))}=1 .
\end{aligned}
$$

Thus $\mathbf{a} \in S$ and $\gamma_{\mathbf{a}}(k)=1$ for each $k \in \mathbf{N}$. 
For $\mathbf{a} \in S$, the sequence $\gamma_{\mathbf{a}}$ is called the correlation of $\mathbf{a}$. It can be extended to the negative integers by setting $\gamma_{\mathbf{a}}(-k)=\overline{\gamma_{\mathbf{a}}(k)}$. It is well known that $\gamma_{\mathbf{a}}$ is positive definite on $\mathbf{Z}$ and thus by the Herglotz-Bochner theorem we have the representation

$$
\gamma_{\mathbf{a}}(n)=\int_{\mathbf{C}_{1}} z^{n} d \lambda_{\mathbf{a}}(z), \quad n \in \mathbf{Z}
$$

where the positive Borel measure $\lambda_{\mathbf{a}}$ is uniquely determined by $\mathbf{a}$; the measure $\lambda_{\mathbf{a}}$ is called the spectral measure corresponding to the sequence $\mathbf{a}$.

If $\nu$ is a bounded (complex-valued) Borel measure on $\mathbf{C}_{1}$, we recall that the Fourier transform $\hat{\nu}$ is given by

$$
\hat{\nu}(k)=\int_{\mathbf{C}_{1}} z^{k} d \nu(z) \quad \text { for } k \in \mathbf{Z} .
$$

In particular, for $\mathbf{a} \in S, \gamma_{\mathbf{a}}=\hat{\lambda}_{\mathbf{a}}$.

The notions of correlation and spectral measure may be generalized as follows (see [7]):

Let $\mathbf{a}=\left(a_{k}\right)$ be a bounded complex sequence. Let $\mathbf{n}=\left(n_{r}\right)$ be an increasing sequence of positive integers such that

$$
\gamma_{\mathbf{a},\left(n_{r}\right)}(k)=\lim _{r} \frac{1}{n_{r}} \sum_{j=0}^{n_{r}-1} a_{j+k} \bar{a}_{j}
$$

exists for each $k \in \mathbf{N}$; we call such a sequence admissible for $\mathbf{a}$. Let $\lambda_{\mathbf{a},\left(n_{r}\right)}$ be the spectral measure corresponding to the correlation function $\gamma_{\mathbf{a},\left(n_{r}\right)}$; then

$$
\hat{\lambda}_{\mathbf{a},\left(n_{r}\right)}(k)=\gamma_{\mathbf{a},\left(n_{r}\right)}(k) .
$$

We write

$$
\Lambda(\mathbf{a})=\left\{\lambda_{\mathbf{a}, \mathbf{n}} \mid \mathbf{n}=\left(n_{r}\right) \text { an admissible sequence for } \mathbf{a}\right\} .
$$

We now review some important and useful approximation results (see [15]).

Let $\mathbf{a}=\left(a_{k}\right)$ be a complex sequence. For each $m \in \mathbf{N}, m \geq 1$, we define the positive Borel measure $\lambda_{\mathbf{a}}^{m}$ on $\mathbf{C}_{1}$ by

$$
\lambda_{\mathbf{a}}^{m}(E)=\int_{E} \frac{1}{m}\left|\sum_{j=0}^{m-1} a_{j} \bar{z}^{j}\right|^{2} d z, \quad E \subset \mathbf{C}_{1} \text { a Borel set. }
$$

With this notation we have

THEOREM $2.1[\mathbf{1 5}]$. Let $\mathbf{a} \in S$. Then the measure $\lambda_{\mathbf{a}}^{m}$ converges weakly to $\lambda_{\mathbf{a}}$ as $m$ tends to infinity.

The corresponding version for bounded complex sequences is

THEOREM $2.2[\mathbf{1 5}]$. Let $\mathbf{a}$ be a bounded complex sequence and let $\mathbf{n}=\left(n_{r}\right)$ be an admissible sequence for $\mathbf{a}$. Then the measure $\lambda_{\mathbf{a}}^{n_{r}}$ converges weakly to $\lambda_{\mathbf{a}, \mathbf{n}}$ as $r$ tends to infinity. have

PROOF. It suffices to show that $\widehat{\lambda_{\mathbf{a}}^{n_{r}}}$ converges pointwise to $\hat{\lambda}_{\mathbf{a}, \mathbf{n}}$. For $k \in \mathbf{Z}$ we

$$
\widehat{\lambda_{\mathbf{a}}^{n_{r}}}(k)=\int_{\mathbf{C}_{1}} z^{k} \frac{1}{n}\left|\sum_{j=0}^{n_{r}-1} a_{j} \bar{z}^{j}\right|^{2} d z=\left\{\begin{array}{cl}
\frac{1}{n_{r}} \sum_{l=0}^{n_{r}-1-k} a_{l+k} \bar{a}_{l} & \text { if } k \geq 0, \\
\frac{1}{n_{r}} \sum_{l=-k}^{n_{r}-1} a_{l+k} \bar{a}_{l} & \text { if } k<0
\end{array}\right.
$$


and hence

$$
\lim _{r} \widehat{\lambda_{\mathbf{a}}^{n_{r}}}(k)=\gamma_{\mathbf{a}, \mathbf{n}}(k)=\hat{\lambda}_{\mathbf{a}, \mathbf{n}}(k)
$$

finishing the proof.

Now let $\mathcal{M}\left(\mathbf{C}_{1}\right)$ be the set of all bounded positive Borel measures on $\mathbf{C}_{1}$. Let $P \in \mathcal{M}\left(\mathbf{C}_{1}\right)$ and $Q \in \mathcal{M}\left(\mathbf{C}_{1}\right)$. Let $\nu \in \mathcal{M}\left(\mathbf{C}_{1}\right)$ be such that $P$ and $Q$ are absolutely continuous with respect to $\nu: P \ll \nu, Q \ll \nu$ (for example, we may take $\nu=$ $P+Q$ ). Then the affinity of $P$ and $Q$ (also known as the Hellinger integral) is defined by

$$
\rho(P, Q)=\int_{\mathbf{C}_{1}}\left(\frac{d P}{d \nu}\right)^{1 / 2}\left(\frac{d Q}{d \nu}\right)^{1 / 2} d \nu .
$$

It is exactly seen that $\rho(P, Q)$ is independent of $\nu$ and that $\rho(P, Q)=0$ if and only if $P$ and $Q$ are mutually singular $(P \perp Q)$.

THEOREM $2.3[\mathbf{1 5}]$. Let $\left(P_{n}\right)$ and $\left(Q_{n}\right)$ be two sequences of elements of $\mathcal{M}\left(\mathbf{C}_{1}\right)$. Let $P \in \mathcal{M}\left(\mathbf{C}_{1}\right)$ and $Q \in \mathcal{M}\left(\mathbf{C}_{1}\right)$. If the sequences $\left(P_{n}\right)$ and $\left(Q_{n}\right)$ converge weakly to $P$ and $Q$ respectively, then

$$
\limsup \rho\left(P_{n}, Q_{n}\right) \leq \rho(P, Q) .
$$

For a proof see $[\mathbf{1 5}, \mathrm{pp} .372-373]$.

Specializing for $\mathbf{a}$ and $\mathbf{b}$ in $S$, namely taking $P=\lambda_{\mathbf{a}}, Q=\lambda_{\mathbf{b}}, P_{n}=\lambda_{\mathbf{a}}^{n}$ and $Q_{n}=\lambda_{\mathbf{b}}^{n}$, the authors obtain the following striking corollary of Theorems 2.1 and 2.3:

Corollary $2.4[\mathbf{1 5}]$. Let $\mathbf{a}, \mathbf{b} \in S$. Then

$$
\underset{n}{\limsup } \frac{1}{n}\left|\sum_{j=0}^{n-1} a_{j} \bar{b}_{j}\right| \leq \rho\left(\lambda_{\mathbf{a}}, \lambda_{\mathbf{b}}\right) .
$$

In particular,

$$
\lim _{n} \frac{1}{n} \sum_{j=0}^{n-1} a_{j} \bar{b}_{j}=0
$$

if $\lambda_{\mathbf{a}}$ and $\lambda_{\mathbf{b}}$ are mutually singular.

The corresponding version for bounded complex sequences is

COROLlaRY $2.5[\mathbf{1 5}]$. Let $\mathbf{a}$ and $\mathbf{b}$ be bounded complex sequences. Then

$$
\underset{n}{\limsup } \frac{1}{n}\left|\sum_{j=0}^{n-1} a_{j} \bar{b}_{j}\right| \leq \sup \rho\left(\lambda_{\mathbf{a}, \mathbf{n}}, \lambda_{\mathbf{b}, \mathbf{m}}\right),
$$

where the supremum in the right-hand side is taken over all $\left(\lambda_{\mathbf{a}, \mathbf{n}}, \lambda_{\mathbf{b}, \mathbf{m}}\right) \in \Lambda(\mathbf{a}) \times$ $\Lambda(\mathbf{b})$.

PROOF. Let $\mathbf{n}=\left(n_{r}\right)$ be an increasing sequence of positive integers such that

$$
\underset{n}{\limsup } \frac{1}{n}\left|\sum_{j=0}^{n-1} a_{j} \bar{b}_{j}\right|=\lim _{r} \frac{1}{n_{r}}\left|\sum_{j=0}^{n_{r}-1} a_{j} \bar{b}_{j}\right| .
$$


By eventually going to a subsequence we may assume that $\mathbf{n}=\left(n_{r}\right)$ is an admissible sequence for both $\mathbf{a}$ and $\mathbf{b}$. By Theorem 2.2,

$$
\lambda_{\mathbf{a}}^{n_{r}} \rightarrow \lambda_{\mathbf{a}, \mathbf{n}} \quad \text { weakly } \quad \text { and } \quad \lambda_{\mathbf{b}}^{n_{r}} \rightarrow \lambda_{\mathbf{b}, \mathbf{n}} \quad \text { weakly, }
$$

and hence, by Theorem 2.3,

$$
\begin{aligned}
& \rho\left(\lambda_{\mathbf{a}, \mathbf{n}}, \lambda_{\mathbf{b}, \mathbf{n}}\right) \geq \limsup _{r} \rho\left(\lambda_{\mathbf{a}}^{n_{r}}, \lambda_{\mathbf{b}}^{n_{r}}\right) \\
& =\underset{r}{\limsup } \frac{1}{n_{r}} \int_{\mathbf{C}_{1}}\left|\sum_{j=0}^{n_{r}-1} a_{j} \bar{z}^{j}\right| \cdot\left|\sum_{k=0}^{n_{r}-1} \bar{b}_{k} z^{k}\right| d z \\
& \geq \underset{r}{\limsup } \frac{1}{n_{r}}\left|\int_{\mathbf{C}_{1}} \sum_{j=0}^{n_{r}-1} \sum_{k=0}^{n_{r}-1} a_{j} \bar{b}_{k} z^{k-j} d z\right| \\
& =\underset{r}{\limsup } \frac{1}{n_{r}}\left|\sum_{j=0}^{n_{r}-1} a_{j} \bar{b}_{j}\right|=\underset{n}{\limsup } \frac{1}{n}\left|\sum_{j=0}^{n-1} a_{j} \bar{b}_{j}\right| .
\end{aligned}
$$

This completes the proof.

COROLlARY 2.6. Let $\mathbf{a}$ and $\mathbf{b}$ be bounded complex sequences. If the spectral measures for $\mathbf{a}$ and $\mathbf{b}$ are mutually singular, that is, if $\lambda_{\mathbf{a}, \mathbf{n}} \perp \lambda_{\mathbf{b}, \mathbf{m}}$ for all $\lambda_{a, \mathbf{n}} \in$ $\Lambda(\mathbf{a})$ and $\lambda_{\mathbf{b}, \mathbf{m}} \in \Lambda(b)$, then

$$
\lim _{n} \frac{1}{n} \sum_{j=0}^{n-1} a_{j} \bar{b}_{j}=0 .
$$

Specializing Corollary 2.4 still further (take $b_{j}=z^{j}$ for $z \in \mathbf{C}_{1}$ ) the authors obtain the classical result of Wiener and Wintner [40] in its full generality; see also Bertrandias [6, p. 25, and 37]:

COROllary 2.7 (Wiener AND Wintner). Let $\mathbf{a} \in S$. Then for each $z \in \mathbf{C}_{1}$ we have

$$
\underset{n}{\limsup } \frac{1}{n}\left|\sum_{j=0}^{n-1} a_{j} \bar{z}^{j}\right| \leq\left[\lambda_{a}(\{z\})\right]^{1 / 2} .
$$

From Corollary 2.7 we obtain the classical ergodic result of Wiener and Wintner [40]; see also [37]:

THEOREM 2.8 (WIENER AND WintNer). Let $T \in C$ and let $f \in L^{1}(\Omega)$. Then there exists a set $\Omega_{f}^{\prime} \subset \Omega$ of probability one such that for each $\omega \in \Omega_{f}^{\prime}$

$$
\lim _{n} \frac{1}{n} \sum_{j=0}^{n-1} z^{j} f\left(T^{j} \omega\right) \quad \text { exists }
$$

for all $z \in \mathbf{C}_{1}$. Furthermore, if $T$ is weakly mixing, then the above limit is zero for all $z \neq 1$.

Since the proof is very similar to that of Theorem 3.15 and Corollary 3.16, we omit it.

For future reference we also state the following lemma whose proof is straightforward. 
LEMmA 2.9. Let $\lambda \in \mathcal{M}\left(\mathbf{C}_{1}\right)$. Let $H$ be a Hilbert space and let $E(\cdot)$ be a spectral measure on $\mathbf{C}_{1}$ whose values are orthogonal projections on $H$. For each $f \in H$ let $\mu_{f}$ be the positive measure on $\mathbf{C}_{1}$ defined by $\mu_{f}(B)=\langle E(B) f, f\rangle, B \subset \mathbf{C}_{1}$ a Borel set. Then we have the orthogonal (Lebesgue type) decomposition of $H$ relative to $\lambda$ :

$$
H=H_{1} \oplus H_{2},
$$

where

$$
H_{1}=\left\{f \in H \mid \mu_{f} \ll \lambda\right\}, \quad H_{2}=\left\{f \in H \mid \mu_{f} \perp \lambda\right\}
$$

and

(i) $H_{1}$ (respectively $\mathrm{H}_{2}$ ) is a closed vector space invariant under each $E(B)$,

(ii) the set $G$ of all $f \in H_{1}$ for which $\mu_{f}=g d \lambda$ with $0 \leq g, g$ bounded, is dense in $H_{1}$.

3. Bounded Besicovitch sequences as good universal weights. Let $\mathfrak{H}_{0}$ be the complex vector space spanned by the set $\left\{\mathbf{a} \mid\right.$ for some $z \in \mathbf{C}_{1}, a_{k}=z^{k}$ for all $k \in \mathbf{N}\}$. The elements of $\mathfrak{H}_{0}$ are the trigonometric polynomials $P=(P(k))$, where $P(k)=\sum_{\alpha} c_{\alpha} z_{\alpha}^{k}$ (here $\left|z_{\alpha}\right|=1$ and the sum is finite).

Let $\mathbf{b}=\left(b_{k}\right)$ be a complex sequence. We say that $\mathbf{b}$ has a mean if $\lim _{n} \frac{1}{n} \sum_{j=0}^{n-1} b_{j}$ exists.

We begin with the following elementary preliminaries $[\mathbf{2}]$.

REMARK. Let $\mathbf{a}(p)$ and $\mathbf{a}$ be complex sequences such that each $\mathbf{a}(p)$ has a mean. Suppose that $\|\mathbf{a}(p)-\mathbf{a}\|_{1} \rightarrow 0$ as $p \rightarrow \infty$. Then a has a mean.

LEMMA 3.1. Let $1 \leq p<\infty, 1<q \leq \infty$ be such that $1 / p+1 / q=1$. Let $\mathbf{b}(n), \mathbf{b}, \mathbf{c}(n)$, c be complex sequences such that $\mathbf{b}(n) \in l(p), \mathbf{b} \in l(p), \mathbf{c}(n) \in l(q)$, $\mathbf{c} \in l(q)$ and suppose that

(i) $\|\mathbf{b}(n)-\mathbf{b}\|_{p} \rightarrow 0,\|\mathbf{c}(n)-\mathbf{c}\|_{q} \rightarrow 0$ as $n \rightarrow \infty$;

(ii) the sequence $\mathbf{b}(n) \mathbf{c}(n)$ has a mean for each $n$.

Then the sequence bc has a mean.

PROOF. It suffices to note that Hölder's inequality holds and therefore

$$
\begin{aligned}
\|\mathbf{b} \mathbf{c}-\mathbf{b}(n) \mathbf{c}(n)\|_{1} & =\|\mathbf{b} \mathbf{c}-\mathbf{b} \mathbf{c}(n)+\mathbf{b} \mathbf{c}(n)-\mathbf{b}(n) \mathbf{c}(n)\|_{1} \\
& \leq\|\mathbf{b}\|_{p}\|\mathbf{c}-\mathbf{c}(n)\|_{q}+\|\mathbf{b}-\mathbf{b}(n)\|_{p}\|\mathbf{c}(n)\|_{q} .
\end{aligned}
$$

We now recall $[8$, Chapter II].

Definition 3.2. Let $1 \leq p<\infty$. A function $f: \mathbf{N} \rightarrow \mathbf{C}$ belongs to the Besicovitch class $B(p)$ if for each $\varepsilon>0$ there exists a trigonometric polynomial $P=P_{\varepsilon}$ such that

$$
\|f-P\|_{p}^{p}=\limsup _{n} \frac{1}{n} \sum_{k=0}^{n-1}|f(k)-P(k)|^{p} \leq \varepsilon
$$

that is, $f \in B(p)$ if and only if $f$ belongs to the \|\|$_{p}$-closure of $\varkappa_{0}$ in $l(p)$.

DEFINITION 3.3. A function $f: \mathbf{N} \rightarrow \mathbf{C}$ is called a bounded Besicovitch function if $f \in B(1) \cap l(\infty)$.

LEMMA 3.4. (1) Let $1 \leq p<\infty$. For $f \in B(p)$ we have

$$
\lim _{n} \frac{1}{n} \sum_{k=0}^{n-1} f(k) \bar{z}^{k} \quad \text { exists for all } z \in \mathbf{C}_{1} \text {. }
$$


(2) For each $f, g \in B(1) \cap l(\infty)$, the limit

$$
\langle f, g\rangle=\lim _{n} \frac{1}{n} \sum_{k=0}^{n-1} f(k) \overline{g(k)}
$$

exists.

(3) For $f, g \in B(2)$,

$$
\langle f, g\rangle=\lim _{n} \frac{1}{n} \sum_{k=0}^{n-1} f(k) \overline{g(k)}
$$

exists and defines an inner space structure. In particular, we have $|\langle f, g\rangle| \leq$ $\|f\|_{2}\|g\|_{2}$ (Cauchy-Schwartz Inequality) for all $f, g \in B(2)$.

PROOF. (1) It suffices to note that the limit in (1) exists for every $f \in \not_{0}$ and to use Lemma 3.1.

(2) By a two-step approximation argument; first show, using Lemma 3.1, that the limit defining $\langle f, g\rangle$ exists whenever $f \in B(1) \cap l(\infty), g \in \mathcal{H}_{0}$.

(3) Again by approximation (using Lemma 3.1).

EXAMPLES. We recall several examples of classes of bounded Besicovitch functions:

(1) The trigonometric polynomials.

(2) The Fourier coefficients of a complex Borel measure on the unit circle. Let $\lambda$ be a complex-valued Borel measure on $\mathbf{C}_{1}$ and let

$$
\hat{\lambda}(j)=\int_{\mathbf{C}_{1}} z^{j} d \lambda(z) \quad \text { for } j \in \mathbf{N} .
$$

Then $(\hat{\lambda}(j))_{j \in \mathbf{N}}$ is bounded Besicovitch. To see this it suffices to decompose $\lambda$ into its continuous and its discrete part, $\lambda=\lambda_{c}+\lambda_{d}$. Note that

$$
\lambda_{d}=\sum_{k} \alpha_{k} \varepsilon_{z_{k}}, \quad \sum_{k}\left|\alpha_{k}\right|<\infty
$$

and thus

$$
\hat{\lambda}_{d}(j)=\sum_{k} \alpha_{k} z_{k}^{j} \quad \text { for each } j \in \mathbf{N}
$$

is a uniform limit of trigonometric polynomials. As for the continuous part, by Wiener's classical theorem (see, for instance, [38, pp. 43-44; 49]),

$$
\frac{1}{n} \sum_{j=0}^{n-1}\left|\hat{\lambda}_{c}(j)\right| \rightarrow 0 .
$$

(3) Almost periodic functions in the sense of Bohr, Weyl, and Eberlein. A function $f: \mathbf{N} \rightarrow \mathbf{C}$ is called almost periodic in the sense of Bohr if for each $\varepsilon>0$ there exists a positive integer $L$ such that every interval $J \subset \mathbf{N}$ of length $L$ contains an $\varepsilon$-period, that is, a number $s \in J$ such that

$$
|f(j+s)-f(j)| \leq \varepsilon \quad \text { for all } j \in \mathbf{N} .
$$

(Equivalently, the set of all $\varepsilon$-periods is relatively dense in $\mathbf{N}$.) We denote by $\mathrm{AP}_{B}(\mathbf{N})$ the set of all almost periodic functions in the sense of Bohr. It is not hard to check that if $f \in \operatorname{AP}_{B}(\mathbf{N})$ then $f$ is bounded. Also if $f, g \in \operatorname{AP}_{B}(\mathbf{N})$, then 
$f \pm g$ and $f \cdot g$ also belong to $A P_{B}(\mathbf{N})$. Thus $\mathrm{AP}_{B}(\mathbf{N}) \subset l(\infty)$ and $\mathrm{AP}_{B}(\mathbf{N})$ is an algebra.

For $f \in l(\infty)$ and $p \in \mathbf{N}$ we denote by $f^{(p)}$ the translate of $f$ by $p$;

$$
f^{(p)}(j)=f(j+p), \quad j \in \mathbf{N} .
$$

LEMMA 3.5. If $f \in \operatorname{AP}_{B}(\mathbf{N})$, then the set $W(f)=\left\{f^{(a)} \mid a \in \mathbf{N}\right\} \subset l(\infty)$ is relatively compact in $l(\infty)$.

ProOF. Given $\varepsilon>0$ let $L=L(\varepsilon)$ correspond to $\varepsilon$ in the definition of almost periodicity and let $J=\{j, j+1, \ldots, j+L\}$ be any interval of length $L$ contained in $\mathbf{N}$. It is easily seen that every translate $f^{(a)}$ belongs to one of the $\varepsilon$-balls centered at $f^{(p)}, p \in J$. If $a \in J$ there is nothing to prove. If $a<j$, then $\{p-a \mid p \in J\}$ is an interval of length $L$ contained in $\mathbf{N}$, and hence contains an $\varepsilon$-period; also

$$
\left|f^{(a)}(n)-f^{(p)}(n)\right|=|f(a+n)-f(a+(p-a)+n)| .
$$

If $a>j+L$, then $\{a-p \mid p \in J\}$ is an interval of length $L$ contained in $\mathbf{N}$, and thus contains an $\varepsilon$-period; also

$$
\left|f^{(a)}(n)-f^{(p)}(n)\right|=|f(p+(a-p)+n)-f(p+n)| .
$$

This completes the proof.

REMARK. The converse implication in Lemma 3.5 is false. The function defined by $f(0)=0, f(n)=1$ for $n \geq 1$ does not belong to $\mathrm{AP}_{B}(\mathbf{N})$; however, the set $\left\{f^{(a)} \mid a \in \mathbf{N}\right\}$ is clearly relatively compact in $l(\infty)$.

A function $f \in l(\infty)$ is called almost periodic in the sense of Weyl if the set $\left\{f^{(a)} \mid a \in \mathbf{N}\right\}$ is relatively compact in $l(\infty)$. We denote by $\operatorname{AP}_{W}(\mathbf{N})$ the set of all almost periodic functions in the sense of Weyl. It is not hard to check that $\operatorname{AP}_{W}(\mathbf{N})$ is an algebra and that both $\mathrm{AP}_{B}(\mathbf{N})$ and $\mathrm{AP}_{W}(\mathbf{N})$ are closed under uniform convergence. By Lemma 3.5 (and the remark following it) we have

$$
\operatorname{AP}_{B}(\mathbf{N}) \varsubsetneqq \operatorname{AP}_{W}(\mathbf{N}) \text {. }
$$

This is in contrast to the theory of almost periodic functions on $\mathbf{Z}$ for which (with similar definitions) $\mathrm{AP}_{B}(\mathbf{Z})=\mathrm{AP}_{W}(\mathbf{Z})$; see, for instance, [8, pp. 10-12, or 16, pp. 14-16].

Fréchet $[\mathbf{2 1}]$ gave a beautiful characterization for the functions $f \in \mathrm{AP}_{W}(\mathbf{N})$ (Fréchet calls them asymptotically almost periodic; his definitions and proofs are given for the continuous semigroup $R_{+}=[0,+\infty)$, but they also work in the discrete case $\mathbf{N}$ ), namely,

LeMma $3.6[\mathbf{2 1}, \mathbf{2 2}]$. For a function $f \in l(\infty)$ the following assertions are equivalent:

(i) $f \in \mathrm{AP}_{W}(\mathbf{N})$;

(ii) $f$ admits a decomposition $f=p+\omega$, where $p \in \mathrm{AP}_{B}(\mathbf{N})$ and $\lim _{n \rightarrow+\infty} \omega(n)$ $=0$ (this decomposition is unique).

A function $f \in l(\infty)$ is called weakly almost periodic--in the sense of Eberlein (see $[\mathbf{2 4}, \mathbf{1 8}]$--if the set $W(f)=\left\{f^{(a)} \mid a \in \mathbf{N}\right\}$ is weakly relatively compact in $l(\infty)$ (i.e. $W(f)$ is relatively compact in $l(\infty)$ endowed with the weak topology $\left.\sigma\left(l(\infty),(l(\infty))^{\prime}\right)\right)$. We denote by $\mathrm{AP}_{w}(\mathbf{N})$ the set of all weakly almost periodic functions. By analogy with Lemma 3.6 Eberlein gave the following characterization for functions $f \in \mathrm{AP}_{w}(\mathbf{N})$ : 
LEMMA $3.7[\mathbf{1 9}]$. For a function $f \in l(\infty)$ the following assertions are equivalent:

(i) $f \in \mathrm{AP}_{w}(\mathbf{N})$;

(ii) $f$ admits a decomposition $f=p+\omega$, where $p \in \mathrm{AP}_{B}(\mathbf{N})$ and

$$
\lim _{n \rightarrow \infty} \frac{1}{n} \sum_{j=0}^{n-1}|\omega(k+j)|=0 \quad \text { uniformly in } k
$$

(this decomposition is unique).

From Lemma 3.7 we easily deduce

COROLlaRY 3.8. The set $\mathrm{AP}_{w}(\mathbf{N})$ is an algebra.

It is also clear that

$$
\mathrm{AP}_{B}(\mathbf{N}) \varsubsetneqq \mathrm{AP}_{W}(\mathbf{N}) \varsubsetneqq \mathrm{AP}_{w}(\mathbf{N})
$$

We now recall the mean properties of almost periodic functions:

LEMMA 3.9. Let $T: l(\infty) \rightarrow l(\infty)$ be the shift operator $T g=g^{(1)} ; T$ is a contraction in $l(\infty)$. Let $f \in \mathrm{AP}_{w}(\mathbf{N})$. Then:

(1) $\overline{c(W(f))}=$ the closed convex hull of $\left\{T^{k} f \mid k \in \mathbf{N}\right\}$ in the weak topology $\sigma\left(l(\infty),(l(\infty))^{\prime}\right)$ is compact.

(2)

$$
\lim _{n \rightarrow \infty} \frac{1}{n} \sum_{j=0}^{n-1} f(k+j)=M(f)
$$

exists uniformly in $k$ and is a constant called the mean of $f$.

(3) For each $k \in \mathbf{N}$

$$
\lim _{n \rightarrow \infty} \frac{1}{n} \sum_{j=0}^{n-1} \bar{f}(j) f(j+k)=M\left[\bar{f} \cdot f^{(k)}\right]
$$

exists.

PROOF. (1) follows from the classical result that in a Banach space the closed convex hull of a weakly compact subset is itself weakly compact.

(2) Let

$$
A(n) f=\frac{1}{n} \sum_{j=0}^{n-1} T^{j} f \text { for } n \geq 1 .
$$

Then $A(n) f \in c(W(f))$ for all $n$ and $T^{n} f / n \rightarrow 0$ in $l(\infty)$. By the Mean Ergodic Theorem (see [17, pp. 661-662, or 18]) $\lim _{n} A(n) f=u$ exists in $l(\infty)$. Since $\lim _{n} A(n) f=\lim _{n} T A(n) f$, the limit function $u$ is invariant under $T$ and hence is a constant which we denote by $M(f)$ and call the mean of $f$. It follows that

$$
\frac{1}{n} \sum_{j=0}^{n-1} f(k+j) \rightarrow M(f) \quad \text { uniformly in } k \text {. }
$$

(3) follows from (2) if we recall (see Corollary 3.8) that $\mathrm{AP}_{w}(\mathrm{~N})$ is an algebra, closed under complex conjugation and invariant under the shift operator, $T\left(\operatorname{AP}_{w}(\mathbf{N})\right) \subset \operatorname{AP}_{w}(\mathbf{N})$.

Note. We shall sometimes write $M(f(j))$ instead of $M(f)$ when we want to emphasize the sequence $(f(j))$. 
LEMMA 3.10. If $f \in \mathrm{AP}_{B}(\mathbf{N}), f \geq 0$ and $M(f)=0$, then $f$ is identically zero.

PROOF. since $\frac{1}{n} \sum_{j=0}^{n-1} f(j) \rightarrow 0$, by the characterization of strong Cesaro convergence for bounded sequences (see $[38$, p. 43]) there is a set $A \subset \mathbf{N}$, of density zero, $d(A)=0$, such that

$$
\lim _{j \notin A} f(j)=0 .
$$

Suppose $f(l)=\alpha>0$. The set $P$ of $(\alpha / 3)$-periods of $f$ is relatively dense, so there is $K$ such that every interval of length $K$ contains some element of $P$. Thus $l d(P+l) \geq$ $1 / K$ and hence there are infinitely many elements belonging to $(P+l) \cap A^{c}$, say, $p_{1}+l, p_{2}+l, \ldots, p_{k}+l, \ldots$ and $\lim _{k} f\left(p_{k}+l\right)=0$. For $k$ large enough we have $\left|f\left(p_{k}+l\right)\right| \leq \alpha / 3$ but also

$$
\left|f(l)-f\left(p_{k}+l\right)\right| \leq \alpha / 3,
$$

whence $|f(l)| \leq \alpha / 3+\alpha / 3=2 \alpha / 3$, a contradiction.

The Fundamental Theorems in the theory of almost periodic functions, namely: (I) approximation in the mean of order $2, M\left[|\cdot|^{2}\right]$, by trigonometric polynomials, and (II) uniform approximation by trigonometric polynomials, hold for all $f \in \mathrm{AP}_{B}(\mathbf{N})$. These can be proven directly by methods analogous to the classical ones $[\mathbf{1 2}]$. Alternatively, one can remark first that every $f \in \mathrm{AP}_{B}(\mathbf{N})$ admits an extension to $R_{+}=[0,+\infty), \tilde{f}$, which is almost periodic in the sense of Bohr on the half-line $R_{+}$, i.e. $\tilde{f} \in \operatorname{AP}_{B}\left(R_{+}\right)$(extend $f$ by linearity on the intervals $[n, n+1)$ ). One then observes that the proofs of the fundamental theorems hold if we replace $R$ by $R_{+}$, i.e. the fundamental theorems hold for all $f \in \mathrm{AP}_{B}\left(R_{+}\right)$. (See [8, pp. 21-31]; here Fundamental Theorem (II) is derived from Fundamental Theorem (I) using $\mathrm{H}$. Weyl's method.)

REMARK. The case of the half-line $R_{+}$(or $\mathbf{N}$ ) can also be reduced to the case of $R$ (or $\mathbf{Z}$ ) as follows: If $f \in \mathrm{AP}_{B}\left(R_{+}\right)$, let $s_{n}$ be $(1 / n)$-periods such that $s_{n} \rightarrow+\infty$ monotonically and set

$$
\tilde{f}(x)=\lim _{n} f\left(x+s_{n}\right) \quad \text { for } x \in R .
$$

For each $x \in R, f\left(x+s_{n}\right)$ is defined for all $n$ sufficiently large and $\left(f\left(x+s_{n}\right)\right)$ is a Cauchy sequence in $R$. (It suffices to observe that $s_{n}-s_{m}$ is a $(1 / n+1 / m)$-period for $f$ if $n>m$; letting $a=s_{n}-s_{m}$ we have for $y \in R_{+}$

$$
\begin{aligned}
\left|f\left(y+\left(s_{n}-s_{m}\right)\right)-f(y)\right| & \leq\left|f(y+a)-f\left(y+a+s_{m}\right)\right|+\left|f\left(y+s_{n}\right)-f(y)\right| \\
& \leq 1 / m+1 / n .)
\end{aligned}
$$

It is now easy to check that $\tilde{f}$ extends $f$, that if $a>0$ is an $\varepsilon$-period for $f$, then $a$ and $-a$ are $\varepsilon$-periods for $\tilde{f}$. It follows that $\tilde{f} \in \operatorname{AP}_{B}(R)$.

A different approach to Fundamental Theorem (II) inspired by Fejér summability was given by Bochner. (Note that Fundamental Theorem (I) is an immediate consequence of Fundamental Theorem (II).)

In the case of a continuous purely periodic function of period $\beta$ on the real line, the Fejér trigonometric polynomials are given by the "convolution product" of $f$ with the Fejér kernel

$$
\sigma_{n}(x)=M_{t}\left[f(x+t) K_{n}(\beta t)\right],
$$


where "the kernel" $K_{n}(\beta t)$ is defined by the equation

$$
K_{n}(t)=\sum_{|\nu|<n}\left(1-\frac{|\nu|}{n}\right) e^{-i \nu t}=\frac{1}{n}\left(\frac{\sin (n / 2) t}{\sin (t / 2)}\right)^{2} .
$$

This kernel has two important properties: (1) it is nonnegative; (2) its mean value is equal to 1 .

In the theory of almost periodic functions, this simple kernel is replaced by the Bochner-Fejér kernel (which is, in fact, a finite product of Fejér kernels):

$$
\begin{aligned}
& K_{\substack{n_{1}, n_{2}, \ldots, n_{p} \\
\beta_{1}, \beta_{2}, \ldots, \beta_{p}}}(t)=K_{n_{1}}\left(\beta_{1} t\right) K_{n_{2}}\left(\beta_{2} t\right) \cdots K_{n_{p}}\left(\beta_{p} t\right) \\
& =\sum_{\substack{\left|\nu_{1}\right|<n_{1} \\
\left|\nu_{p}\right|<n_{p}}}\left(1-\frac{\left|\nu_{1}\right|}{n_{1}}\right)\left(1-\frac{\left|\nu_{2}\right|}{n_{2}}\right) \cdots\left(1-\frac{\left|\nu_{p}\right|}{n_{p}}\right) e^{-i\left(\nu_{1} \beta_{1}+\nu_{2} \beta_{2}+\cdots+\nu_{p} \beta_{p}\right) t},
\end{aligned}
$$

where $\beta_{1}, \beta_{2}, \ldots, \beta_{p}$ are real linearly independent numbers and $n_{1}, n_{2}, \ldots, n_{p}$ are positive integers. This kernel inherits the basic properties of the Fejér kernel: (1) it is nonnegative; (2) its mean value is equal to 1 (the constant term being 1 on account of the linear independence of the $\beta$ 's). Using the matrix notation

$$
B=\left(\begin{array}{c}
n_{1}, n_{2}, \ldots, n_{p} \\
\beta_{1}, \beta_{2}, \ldots, \beta_{p}
\end{array}\right),
$$

the Bochner-Fejér kernel in our discrete case becomes:

$$
K_{B}(k)=K_{n_{1}}\left(\beta_{1} k\right) K_{n_{2}}\left(\beta_{2} k\right) \cdots K_{n_{p}}\left(\beta_{p} k\right), \quad k \in \mathbf{N} .
$$

Clearly $K_{B}=\left(K_{B}(k)\right)$ belongs to $\nvdash_{0}$. For $f=(f(k))$ belonging to $\operatorname{AP}_{w}(\mathbf{N})$ (see Corollary 3.8), or $f$ belonging to $B(1$ ) (see Lemma 3.4) we can form the corresponding Bochner-Fejér polynomial

$$
\sigma_{B}^{f}(k)=M\left[f(k+j) K_{B}(j)\right] .
$$

With the above notation we have

Fundamental Theorem II. Let $f \in \mathrm{AP}_{B}(\mathbf{N})$. There exists then a sequence of Bochner-Fejér polynomials $\left(\sigma_{B_{n}}^{f}\right)$ such that

$$
\left\|\sigma_{B_{n}}^{f}-f\right\|_{\infty} \rightarrow 0 \text { as } n \rightarrow \infty .
$$

For a proof see $[8$, pp. 47-50].

LemMA 3.11 . (1) Let $1 \leq p<\infty$. Let $f \in B(p)$. Then:

(a) For any Bochner-Fejér polynomial $\sigma_{B}^{f}$ we have $\left\|\sigma_{B}^{f}\right\|_{p} \leq\|f\|_{p}$.

(b) There exists a sequence of Bochner-Fejér polynomials $\left(\sigma_{B_{n}}^{f}\right)$ such that

$$
\left\|\sigma_{B_{n}}^{f}-f\right\|_{p} \rightarrow 0 \text { as } n \rightarrow \infty .
$$

(c) If, in addition, $f$ is also bounded, then for any Bochner-Fejér polynomial $\sigma_{B}^{f}$ we have $\left\|\sigma_{B}^{f}\right\|_{\infty} \leq\|f\|_{\infty}$.

(2) For bounded functions all the Besicovitch classes $B(p)$ coincide, that is

$$
B(1) \cap l(\infty)=B(p) \cap l(\infty) \text { for } 1 \leq p<\infty .
$$


PROOF. For the proof of $(1)(\mathrm{a})$ and (b) see [8, pp. 107-109].

(1)(c) follows immediately from the definition of $\sigma_{B}^{f}$ and the basic properties of the Bochner-Fejér kernel (it is nonnegative and its mean value is equal to 1 ).

(2) is an immediate consequence of $(1)(b)$ and (c).

Combining Lemmas 3.5, 3.6, 3.7, 3.11 and Fundamental Theorem II, we get

LEMMA 3.12. We have

$$
\mathrm{AP}_{B}(\mathbf{N}) \subset \mathrm{AP}_{W}(\mathbf{N}) \subset \mathrm{AP}_{w}(\mathbf{N}) \subset B(2) \cap l(\infty)=B(1) \cap l(\infty) .
$$

We need one more observation which we state in the form of a lemma:

LEMMA 3.13. For a sequence $f=(f(j))$ belonging to $S \cap l(\infty)$ the following assertions are equivalent:

(i) The spectral measure $\sigma_{f}$ corresponding to $f$ is discrete.

(ii) The correlation of $f, \gamma_{f}$, is almost periodic in the sense of Bohr, i.e. $\gamma_{f} \in$ $\mathrm{AP}_{B}(\mathbf{N})$.

If (i) $\equiv$ (ii) holds, then

$$
\sigma_{f}=\sum_{n=1}^{\infty} A_{n} \varepsilon_{z_{n}} \quad\left(A_{n}>0, z_{n} \in \mathbf{C}_{1}\right)
$$

and

$$
\gamma_{f}(k)=\sum_{n=1}^{\infty} A_{n} z_{n}^{k}
$$

the convergence being uniform in $k$.

ProOF. (i) $\Rightarrow$ (ii). By (i) we have

$$
\sigma_{f}=\sum_{n=1}^{\infty} A_{n} \varepsilon_{z_{n}} \quad\left(A_{n}>0, z_{n} \in \mathbf{C}_{1}\right) ;
$$

here and in what follows $\varepsilon_{\lambda}=$ Dirac measure at $\lambda$. It follows that

$$
\gamma_{f}(k)=\int_{\mathbf{C}_{1}} z^{k} d \sigma_{f}(z)=\sum_{n=1}^{\infty} A_{n} z_{n}^{k},
$$

the convergence being uniform in $k$. Hence $\gamma_{f} \in \mathrm{AP}_{B}(\mathbf{N})$.

(ii) $\Rightarrow$ (i). Since

$$
\gamma_{f}(k)=\int_{\mathbf{C}_{1}} z^{k} d \sigma_{f}(z) \quad \text { for } k \in \mathbf{N},
$$

it follows that

$$
M\left[\gamma_{f}(j) \bar{z}^{j}\right]=\sigma_{f}(\{z\}) \geq 0
$$

for each $z \in \mathbf{C}_{1}$. Thus $\gamma_{f}$ is an almost periodic function in the sense of Bohr having nonnegative Bohr-Fourier coefficients. By a classical result (see $[8$, p. 52]), it follows that the Bohr-Fourier series of $\gamma_{f}$ converges absolutely and uniformly, that is, if $\gamma_{f}(k) \sim \sum_{n=1}^{\infty} A_{n} z_{n}^{k}$, then $\sum_{n=1}^{\infty}\left|A_{n}\right|=\sum_{n=1}^{\infty} A_{n}<\infty$ and $\gamma_{f}(k)=\sum_{n=1}^{\infty} A_{n} z_{n}^{k}$ uniformly in $k$.

By the uniqueness in the Herglotz-Bochner theorem we conclude that

$$
\sigma_{f}=\sum_{n=1}^{\infty} A_{n} \varepsilon_{z_{n}}
$$


i.e. the spectral measure $\sigma_{f}$ is discrete. This concludes the proof.

Finally we review the following important theorem due to Wiener and Wintner.

THEOREM $3.14[\mathbf{4 1}]$. A sequence $f=(f(j))$ belonging to $S \cap l(\infty)$ is bounded Besicovitch, i.e. $f \in B(2) \cap l(\infty)$, if and only if $f$ satisfies the following conditions:

(1) The spectral measure $\sigma=\sigma_{f}$ corresponding to $f$ is discrete.

(2) The amplitude $\Gamma(z)=M\left[f(j) \bar{z}^{j}\right]=\lim _{n} \frac{1}{n} \sum_{j=0}^{n-1} f(j) \bar{z}^{j}$ exists for each $z \in$ $\mathbf{C}_{1}$.

(3) $\sigma(\{z\})=|\Gamma(z)|^{2}$ for all $z \in \mathbf{C}_{1}$.

ProOF. Assume first that $f \in B(2) \cap l(\infty)$. The existence of the correlation of $f$ and of $\Gamma$ follows from Lemma 3.4. It remains to check (1) and (3). We divide the proof into two steps.

(I) If $p$ is a trigonometric polynomial $p(k)=\sum_{\alpha} c_{\alpha} z_{\alpha}^{k}$, then a direct calculation shows that the correlation of $p, \gamma_{p}$, is again a trigonometric polynomial

$$
\gamma_{p}(k)=M\left[p^{(k)} \cdot \bar{p}\right]=\sum_{\alpha}\left|c_{\alpha}\right|^{2} z_{\alpha}^{k}
$$

In particular, the spectral measure $\sigma_{p}$ corresponding to $p$ is discrete and is given by $\sigma_{p}=\sum_{\alpha}\left|c_{\alpha}\right|^{2} \varepsilon_{z_{\alpha}}$; that is, $\sigma_{p}(\{z\})=\left|\Gamma_{p}(z)\right|^{2}$ for all $z \in \mathbf{C}_{1}$ (where, of course, $\Gamma_{p}(z)=M\left[p(k) \bar{z}^{k}\right]=$ the corresponding Bohr-Fourier coefficient), or equivalently,

$$
M\left[\gamma_{p}(k) \bar{z}^{k}\right]=\left|M\left[p(k) \cdot \bar{z}^{k}\right]\right|^{2} \text { for all } z \in \mathbf{C}_{1} .
$$

(II) By Lemma 3.11 now let $\left(p_{n}\right)$ be a sequence of Bochner-Fejér polynomials such that

$$
\left\|f-p_{n}\right\|_{2}^{2}=M\left[\left|f-p_{n}\right|^{2}\right] \rightarrow 0
$$

and

$$
\left\|p_{n}\right\|_{\infty} \leq\|f\|_{\infty} \text { for all } n \in \mathbf{N}
$$

It follows easily that

$$
\gamma_{f}(k)=M\left[f^{(k)} \cdot \bar{f}\right]=\lim _{n} M\left[p_{n}^{(k)} \cdot \bar{p}_{n}\right]=\lim _{n} \gamma_{p_{n}}(k)
$$

uniformly in $k$. Thus $\gamma_{f}$ being a uniform limit of trigonometric polynomials is almost periodic in the sense of Bohr, i.e. $\gamma_{f} \in \mathrm{AP}_{B}(\mathbf{N})$. By Lemma 3.13 condition $(1)$ is satisfied. Since the identity $(*)$ above holds for each $p_{n}$ and since the passage to the limit is legitimate, condition (3) is also satisfied.

Assume now that $f \in S \cap l(\infty)$ satisfies conditions (1), (2) and (3) of the theorem. By (1) and Lemma 3.13 there exist a sequence $z_{1}, z_{2}, \ldots, z_{n}, \ldots$ of distinct numbers of $\mathbf{C}_{1}$, and a sequence $A_{1}, A_{2}, \ldots, A_{n}, \ldots\left(A_{n}>0\right)$ such that

$$
\sigma=\sigma_{f}=\sum_{n=1}^{\infty} A_{n} \varepsilon_{z_{n}}, \quad \sum_{n=1}^{\infty} A_{n}<+\infty
$$

and

$$
\gamma(k)=\gamma_{f}(k)=\sum_{n=1}^{\infty} A_{n} z_{n}^{k}
$$


the convergence being uniform in $k$. By conditions (2) and (3), $\Gamma(z)=\Gamma_{f}(z) \neq 0$ only for $z \in\left\{z_{1}, z_{2}, \ldots, z_{n}, \ldots\right\}$. Let $a_{n}=\Gamma\left(z_{n}\right)\left(=M\left[f(k) \bar{z}_{n}^{k}\right]\right)$; then $A_{n}=\left|a_{n}\right|^{2}$. Define now $f_{N}=\left(f_{N}(k)\right)$ by

$$
f_{N}(k)=f(k)-\sum_{n \leq N} a_{n} z_{n}^{k}
$$

To show that $f \in B(2)$ it suffices to show that

$$
\left\|f_{N}\right\|_{2}^{2}=M\left[\left|f(k)-\sum_{n \leq N} a_{n} z_{n}^{k}\right|^{2}\right] \rightarrow 0 \quad \text { as } N \rightarrow \infty .
$$

It is clear that $f_{N}$ has a correlation and a direct calculation shows that it is given by

$$
\gamma_{N}(k)=\gamma(k)-\sum_{n \leq N}\left|a_{n}\right|^{2} z_{n}^{k}
$$

It is also easily checked that the spectral measure $\sigma_{N}$ corresponding to $f_{N}$ is given by $\sigma_{N}=\sum_{n>N} A_{n} \varepsilon_{z_{n}} ;$ in fact, for each $k \in \mathbf{N}$,

$$
\int_{\mathbf{C}_{1}} z^{k} d \sigma_{N}(z)=\sum_{n>N} A_{n} z_{n}^{k}=\gamma(k)-\sum_{n \leq N} A_{n} z_{n}^{k}=\gamma_{N}(k)
$$

But then

$$
\left\|f_{N}\right\|_{2}^{2}=M\left[\left|f_{N}\right|^{2}\right]=\gamma_{N}(0)=\int_{\mathbf{C}_{1}} 1 d \sigma_{N}(z)=\sum_{n>N} A_{n} \rightarrow 0
$$

as $N \rightarrow \infty$. This finishes the proof.

REMARK. The class of sequences $f=(f(j))$ belonging to $S \cap l(\infty)$ and satisfying only conditions (1) and (2) of Theorem 3.14, called $\mathcal{M}^{1}$-almost periodic by Bertrandias (see [7, pp. 69-73]), is strictly larger than the class of bounded Besicovitch sequences.

EXAMPLE. Let $f(j)=(-1)^{[\log j]}$ (here and in what follows $[x]$ denotes the integral part of $x$, i.e. the largest integer $k \leq x)$. It is easy to see that the sequence $f=(f(j))$ has a correlation $\gamma=\gamma_{f}$ and that $\gamma(p)=1$ for all $p \in \mathbf{N}$. Thus the spectral measure $\sigma=\sigma_{f}$ corresponding to $f$ is Dirac measure at 1 , that is, $\sigma$ is discrete. It is also not hard to check that the amplitude $\Gamma(z)=\lim _{n} \frac{1}{n} \sum_{j=0}^{n-1} f(j) \bar{z}^{j}$ exists and $=0$ for all $z \in \mathbf{C}_{1}$. In particular, $\sigma(\{1\})=1$ while $|\Gamma(1)|^{2}=0$. Thus the sequence $f=(f(j))$ satisfies conditions (1) and (2) of Theorem 3.14, but fails to satisfy condition (3).

THEOREM 3.15. Let $D$ be the set of all $a \in S \cap l(\infty)$ satisfying the following conditions:

(1) The spectral measure $\sigma_{\mathbf{a}}$ corresponding to $\mathbf{a}$ is discrete.

(2) The amplitude $\lim _{n} \frac{1}{n} \sum_{j=0}^{n-1} a_{j} \bar{z}^{j}$ exists for all $z \in \mathbf{C}_{1}$.

Then every $\mathbf{a} \in D$ is a good universal weight. In fact more is true. Let $T \in \mathcal{C}$ ergodic. For each $f \in L^{1}(\Omega)$ there exists a set $\Omega_{f} \subset \Omega$ of probability one such that

$$
\lim _{n} \frac{1}{n} \sum_{j=0}^{n-1} a_{j} \overline{f\left(T^{j} \omega\right)} \text { exists }
$$

for any $\mathbf{a} \in D$ and any $\omega \in \Omega_{f}$. 
Proof. Let $T \in C$ ergodic. We divide the proof into two parts:

(I) Let $g \in L^{2}(\Omega)$ and let $\Omega^{(g)}$ be the set of all $\omega \in \Omega$ for which the sequence $\mathbf{b}=\left(g\left(T^{k} \omega\right)\right)$ has a correlation given by the spectral measure of $U_{T}$, via

$$
\lim _{n} \frac{1}{n} \sum_{j=0}^{n-1} g\left(T^{j+k} \omega\right) \bar{g}\left(T^{j} \omega\right)=\left\langle U_{T}^{k} g, g\right\rangle=\int_{\mathbf{C}_{1}} \lambda^{k} d\langle E(\lambda) g, g\rangle .
$$

By the individual Ergodic Theorem, $\mu\left(\Omega^{(g)}\right)=1$. Now let $V_{1}$ be the linear space spanned by the eigenfunctions of $U_{T}$ and let

$$
V_{2}=\left\{f \in L^{2}(\Omega) \mid\langle E(\cdot) f, f\rangle \text { is continuous }\right\} .
$$

Clearly $V=V_{1}+V_{2}$ is dense in $L^{2}(\Omega)$. By assumption (2) it is clear that if $\mathbf{a} \in D$

$$
\lim _{n} \frac{1}{n} \sum a_{j} \bar{g}\left(T^{j} \omega\right) \quad \text { exists for all } \omega \in \Omega
$$

if $g$ is an eigenfunction of $U_{T}$. Now let $g \in V_{2}$; then for each $\omega \in \Omega^{(g)}$, the spectral measure corresponding to the sequence $\mathbf{b}=\left(g\left(T^{k} \omega\right)\right),\langle E(\cdot) g, g\rangle$, being continuous, is singular with respect to $\sigma_{\mathbf{a}}$, and thus by Corollary 2.4 ,

$$
\lim _{n} \frac{1}{n} \sum_{j=0}^{n-1} a_{j} \bar{g}\left(T^{j} \omega\right)=0
$$

for each $\mathbf{a} \in D$ and $\omega \in \Omega^{(g)}$.

(II) Now let $f \in L^{1}(\Omega)$. Let $g_{p} \in V$ be such that $g_{p} \stackrel{L^{1}}{\rightarrow} f$. By step (I) and the Individual Ergodic Theorem, we can find for each $p$ a set $\Omega_{p} \subset \Omega$ of full measure with the following properties:

$\left(1^{\circ}\right) \omega \in \Omega_{p} \Rightarrow \lim _{n} \frac{1}{n} \sum_{j=0}^{n-1} a_{j} \bar{g}_{p}\left(T^{j} \omega\right)$ exists for all $\mathbf{a} \in D$;

$\left(2^{\circ}\right) \omega \in \Omega_{p} \Rightarrow \lim _{n} \frac{1}{n} \sum_{j=0}^{n-1}\left|f\left(T^{j} \omega\right)-g_{p}\left(T^{j} \omega\right)\right|=\int\left|f-g_{p}\right| d \mu$.

Let $\Omega_{f}=\bigcap_{p=1}^{\infty} \Omega_{p}$. For fixed $\omega \in \Omega_{f}$, let $\mathbf{b}^{*}=\left(f\left(T^{k} \omega\right)\right), \mathbf{b}(p)=\left(g_{p}\left(T^{k} \omega\right)\right)$; note that $\left\|\mathbf{b}^{*}-\mathbf{b}(p)\right\|_{1} \rightarrow 0$ and apply Lemma 3.1 to deduce that

$$
\lim _{n} \frac{1}{n} \sum_{j=0}^{n-1} a_{j} \overline{f\left(T^{j} \omega\right)} \quad \text { exists }
$$

for each $\mathbf{a} \in D$.

An application of Theorem 1.4 concludes the proof.

COROLLARY 3.16. Let $D$ be the set of all $\mathbf{a} \in S \cap l(\infty)$ satisfying conditions (1) and (2) of Theorem 3.15. Let $T \in C$. For each $f \in L^{1}(\Omega)$ there exists a set $\Omega_{f} \subset \Omega$ of probability one such that for any $\omega \in \Omega_{f}$,

$$
\lim _{n} \frac{1}{n} \sum_{j=0}^{n-1} a_{j} \overline{f\left(T^{j} \omega\right)} \quad \text { exists }
$$

for all $\mathbf{a} \in D$, in particular for all bounded Besicovitch sequences.

PROOF. Use "decomposition into ergodic parts". 
REMARKS. (1) The main theorem in $[\mathbf{1 0}]$ is a special case of Theorem 3.15: the case when $T$ is weakly mixing and a an arbitrary $p$-sequence.

Indeed it was recently shown by Losert in $[\mathbf{3 1}]$ that every $p$-function is a bounded Besicovitch function and that the inclusion is strict; Losert [31] also obtains an intrinsic characterization of $p$-functions in terms of the Fourier transforms of real signed measures on $\mathbf{C}_{1}$, analogous to that of Riemann integrable functions in terms of continuous functions.

(2) Theorem 3.15 and Corollary 3.16 also generalize Theorem 6 in $[\mathbf{3 6}]$ : the class of bounded Besicovitch sequences $B(2) \cap l(\infty)$ is replaced by the larger class $D$, and the set $\Omega_{f}$ of full measure depends only on $f$ but not on the particular sequence $\mathbf{a} \in D$.

(3) The pioneering work on the Pointwise Ergodic Theorem along subsequences (weights) was done by Brunel and Keane [13]. It turns out that the class of uniform sequences introduced by Brunel and Keane is contained in the class of bounded Besicovitch sequences (it is, in fact, already contained in the class of $p$-sequences, as is shown in $[\mathbf{1 0}])$. We sketch the proof below.

We recall the definition of a strictly L-stable (stable in the sense of Lyapunov) system given in $[\mathbf{1 3}]$.

Let $X$ be a compact metric space, and $\phi: X \rightarrow X$ a homeomorphism of $X$.

DEFINITION 3.17. We say that the system $(X, \phi)$ is strictly $L$-stable if: (i) The set $\left\{\phi^{n} \mid n \in \mathbf{Z}\right\}$ is uniformly equicontinuous, and (ii) $X$ possesses a dense orbit, that is, there is an $x \in X$ such that $\left\{\phi^{n} x \mid n \in \mathbf{Z}\right\}$ is dense in $X$.

We have

LEMMA 3.18 (SEE [33 OR 36]). Let $(X, \phi)$ be a strictly L-stable system. Then $(X, \phi)$ is strictly ergodic, that is, there exists a unique $\phi$-invariant (and thus ergodic) probability on the $\sigma$-field $X$ of Borel subsets of $X$, which we denote by $\nu$ and each nonempty open set has positive $\nu$-measure. In particular for every $f \in C(X)$ and $x \in X$,

$$
\lim _{n} \frac{1}{n} \sum_{k=0}^{n-1} f\left(\phi^{k} x\right)=\int_{X} f d \nu .
$$

Throughout the remainder of this section we shall deal with a strictly $L$-stable system and we shall write $(X, \mathcal{X}, \nu, \phi)$ instead of $(X, \phi)$.

THEOREM 3.19. Let $(X, \mathcal{X}, \nu, \phi)$ be a strictly L-stable system. Then we have:

(1) For any $f \in C(X)$ and any $x \in X$, the sequence $\mathbf{u}$ given by $\mathbf{u}=\left(f\left(\phi^{n}(x)\right)\right)$ is almost periodic in the sense of Bohr, i.e. belongs to $\operatorname{AP}_{B}(\mathbf{N})$.

(2) For each $g \in L^{\infty}(X, \mathcal{X}, \nu)$ there exists a set $X^{\prime} \subset X$ of $\nu$-probability one such that for each $x \in X^{\prime}$ the sequence $\mathbf{v}$ given by $\mathbf{v}=\left(g\left(\phi^{n}(x)\right)\right)$ is bounded Besicovitch.

ProOF. (1) By our assumption, the set of functions $\left\{f \circ \phi^{n} \mid n \in \mathbf{Z}\right\}$ is uniformly bounded and uniformly equicontinuous, and thus by Arzela's theorem it is relatively compact for the topology of uniform convergence on $X$. It follows that for any fixed $x \in X$ the sequence $\mathbf{u}=\left(f\left(\phi^{n}(x)\right)\right)_{n \in \mathbf{Z}}$ belongs to $\mathrm{AP}_{W}(\mathbf{Z})=\mathrm{AP}_{B}(\mathbf{Z})$ and hence its restriction to $\mathbf{N}, u$ belongs to $\operatorname{AP}_{B}(\mathbf{N})$.

(2) Let $g \in L^{\infty}(X, \chi, \nu)$. Choose $f_{p} \in C(X)$ such that

$$
f_{p} \stackrel{L^{1}(X, \nu)}{\rightarrow} g .
$$


For each $p$, by the Individual Ergodic Theorem, we can find a set $X_{p} \subset X$ of full measure such that

$$
x \in X_{p} \Rightarrow \lim _{n} \frac{1}{n} \sum_{k=0}^{n-1}\left|f_{p}\left(\phi^{k}(x)\right)-g\left(\phi^{k}(x)\right)\right|=\int_{X}\left|f_{p}-g\right| d \nu .
$$

Let $X^{\prime}=\bigcap_{p=1}^{\infty} X_{p}$. For fixed $x \in X^{\prime}$, let $\mathbf{v}=\left(g\left(\phi^{n}(x)\right)\right)$ and $\mathbf{u}(p)=\left(f_{p}\left(\phi^{n}(x)\right)\right)$; note that

$$
\|\mathbf{v}-\mathbf{u}(p)\|_{1}=\int_{X}\left|g-f_{p}\right| d \nu \rightarrow 0
$$

and that each $\mathbf{u}(p)$ is almost periodic in the sense of Bohr. This concludes the proof.

We recall that a bounded function $f: X \rightarrow R$ is said to be Riemann integrable with respect to $\nu$ if the set $D=\{x \in X \mid f$ is discontinuous at $x\}$ has $\nu$-measure zero.

The following useful characterization of Riemann integrable functions is elementary but not entirely trivial (it makes use of the oscillation functions of $f$,

$$
\operatorname{Osc}_{f}(x)=\inf \left\{\sup _{y, z \in V}|f(y)-f(z)| \mid V \text { a neighborhood of } x\right\}
$$

and of the Urysohn theorem):

LemMA 3.20. Let $f: X \rightarrow R$ be bounded. Then the following assertions are equivalent:

(i) $f$ is Riemann integrable with respect to $\nu$.

(ii) For each $\varepsilon>0$ there are functions $u \in C(X), v \in C(X)$ such that $u \leq f \leq v$ and $\int(v-u) d \nu \leq \varepsilon$.

COROLlaRY 3.21. Let $h: X \rightarrow R$ be Riemann integrable with respect to $\nu$. Let $y \in X$. Let $\mathbf{a}$ be the sequence given by $\mathbf{a}=\left(h\left(\phi^{n}(y)\right)\right)$. Then for each $\varepsilon>0$ there are $\mathbf{u}=\left(u_{n}\right)$ and $\mathbf{v}=\left(v_{n}\right)$ belonging to $\mathrm{AP}_{B}(\mathbf{N})$ such that

$$
u_{n} \leq h\left(\phi^{n}(y)\right) \leq v_{n} \quad \text { for all } n \in \mathbf{N}
$$

and

$$
\|\mathbf{v}-\mathbf{u}\|_{1}=\lim _{n} \frac{1}{n} \sum_{k=0}^{n-1}\left(v_{k}-u_{k}\right) \leq \varepsilon
$$

in particular a is bounded Besicovitch.

Proof. By Lemma 3.20, for each $\varepsilon>0$ there are $f, g \in C(X)$ such that $f \leq$ $h \leq g$ and $\int(g-f) d \nu \leq \varepsilon$. Let $\mathbf{u}=\left(f\left(\phi^{n}(y)\right)\right)$ and $\mathbf{v}=\left(g\left(\phi^{n}(y)\right)\right)$. It suffices to note that $\mathbf{u}$ and $\mathbf{v}$ are almost periodic in the sense of Bohr by Theorem 3.19 and that

by Lemma 3.18 .

$$
\lim _{n} \frac{1}{n} \sum_{k=0}^{n-1}\left(g\left(\phi^{k}(y)\right)-f\left(\phi^{k}(y)\right)\right)=\int(g-f) d \nu \leq \varepsilon
$$

We recall the definition of the Bohr compactification of $\mathbf{Z}$ going back to the fundamental work of Anzai and Kakutani (see [1]). Let $\left(\mathbf{C}_{1}\right)_{d}$ be the unit circle group with the discrete topology. Then $b \mathbf{Z}$, the Bohr compactification of $\mathbf{Z}$, is the dual of $\left(\mathbf{C}_{1}\right)_{d} ; b \mathbf{Z}$ is a compact abelian group and $\mathbf{Z} \subset b \mathbf{Z}$ is dense in $b \mathbf{Z}$. Further 
every $f \in \operatorname{AP}_{B}(\mathbf{Z})=\operatorname{AP}_{W}(\mathbf{Z})$ has a (unique) continuous extension to $b \mathbf{Z}$; in other words, the almost periodic functions on $\mathbf{Z}$ are precisely the restrictions to $\mathbf{Z}$ of the continuous functions on $b \mathbf{Z}$.

We recall also that a topological group $G$ is called monothetic if there is $u \in G$ such that the cyclic group generated by $u,\left\{u^{n} \mid n \in \mathbf{Z}\right\}$, is dense in $G$. A monothetic group is clearly abelian. It is well known that every compact monothetic group is the continuous homomorphic image of $b \mathbf{Z}$, i.e., is isomorphic to a quotient group of $b \mathbf{Z}$.

REMARKS. (1) It is known (see [27]) that any strictly $L$-stable system $(X, \phi)$ is "isomorphic" in the sense of Ergodic Theory [38] with a system $(Y, \psi)$, where $Y$ is a compact metrizable monothetic group and $\psi$ is translation by an element $u \in Y$ generating a dense cyclic group (under this isomorphism the measure $\nu$ gets mapped onto Haar measure measure on $Y$ ).

(2) The correspondence between $\operatorname{AP}_{B}(\mathbf{Z})$ and $C(b \mathbf{Z})$ referred to above can be extended to a bijection between $\tilde{B}(p)$ and $L^{p}(b \mathbf{Z})(1 \leq p \leq \infty)$ which preserves the $p$-norm, the algebraic (addition, multiplication, translation) and order structures $(\tilde{B}(p)$ denotes, of course, the quotient space of $B(p)$ by the space of all $f$ with $\left.\|f\|_{p}=0\right)$. Using this correspondence one may show that in a certain sense a converse to Theorem 3.19(2) also holds, namely: If $\mathbf{v}=\left(v_{n}\right)$ is bounded Besicovitch, there exist a compact metrizable monothetic group $Y, f \in L^{\infty}(Y), u \in G$ generating a dense cyclic group such that $v_{n}=f\left(T^{n} u\right.$ ) (here, of course, $T y=u y$ ) and $u$ is generic for the $T$-invariant algebra $\mathfrak{A}$ generated by $f$ and the continuous functions on $Y$ (i.e., $\frac{1}{n} \sum_{k=0}^{n-1} g\left(T^{k} u\right) \rightarrow \int g d \nu$, for $g \in \mathfrak{A} ; \nu=$ Haar measure). We omit the proof. For further details one may consult $[\mathbf{2 0}]$.

(3) Concerning Corollary 3.21, the special case when $h=1_{A}, A \subset X$ a Jordan set with respect to $\nu$ (that is the boundary $\partial A$ of $A$ has $\nu$-measure zero), leads to the weights $a=\left(1_{A}\left(\phi^{n}(y)\right)\right)$ associated with the so-called "uniform sequences" of Brunel and Keane $[\mathbf{1 3}]$.

We recall that an ergodic automorphism $T \in C$ has discrete spectrum if $L^{2}(\Omega)$ has an orthonormal basis consisting of eigenfunctions of $U_{T}$.

We conclude this section with the following result.

THEOREM 3.22. For $T \in C$ ergodic the following two assertions are equivalent:

(i) $T$ has discrete spectrum.

(ii) If $g \in L^{\infty}(\Omega)$, then for almost every $\omega \in \Omega$, the sequence $\mathbf{v}$ given by $\mathbf{v}=$ $g\left(T^{k}(\omega)\right)$ is bounded Besicovitch.

PROOF. The technique of proof is similar to that used in the proof of Theorems 3.15 and 3.19. For the sake of completeness we include the details.

(i) $\Rightarrow$ (ii). Let $\left\{f_{j}\right\}$ be an orthonormal basis consisting of eigenfunctions of $U_{T}$ and let $V_{0}$ be the linear space spanned by $\left\{f_{j}\right\}$.

Now let $g \in L^{\infty}(\Omega)$. Let $h_{p} \in V_{0}$ be such that

$$
h_{p} \stackrel{L^{1}}{\rightarrow} g .
$$

By the Individual Ergodic Theorem, we can find for each $p$ a set $\Omega_{p} \subset \Omega$ of full measure with the following property:

$$
\omega \in \Omega_{p} \Rightarrow \lim _{n} \frac{1}{n} \sum_{j=0}^{n-1}\left|g\left(T^{j} \omega\right)-h_{p}\left(T^{j} \omega\right)\right|=\int\left|g-h_{p}\right| d \mu .
$$


Let $\Omega_{(g)}=\bigcap_{p=1}^{\infty} \Omega_{p}$. For fixed $\omega \in \Omega_{(g)}$, let $\mathbf{v}=\left(g\left(T^{k} \omega\right)\right)$ and $\mathbf{u}(p)=\left(h_{p}\left(T^{k} \omega\right)\right)$; it is then clear that $\mathbf{u}(p)$ is a trigonometric polynomial and that $\|\mathbf{v}-\mathbf{u}(p)\|_{1} \rightarrow \mathbf{0}$ as $p \rightarrow \infty$. This shows that $\mathbf{v}=\left(g\left(T^{k} \omega\right)\right)$ is bounded Besicovitch.

(ii) $\Rightarrow\left(\right.$ i). Let $V$ be the closed linear space spanned by the eigenfunctions of $U_{T}$ and let $W=V^{\perp}=\left\{f \in L^{2}(\Omega) \mid\langle E(\cdot) f, f\rangle\right.$ is continuous $\}$. By Theorem 5.2 in [2], every $f \in L^{\infty}(\Omega)$ can be written in the form

$$
f=g+h, \quad g \in V \cap L^{\infty}(\Omega), h \in W \cap L^{\infty}(\Omega)
$$

(the orthogonal projection onto $V$ defines an $L^{\infty}$-contraction). Suppose, by contradiction, that $T$ does not have discrete spectrum. Then $W \cap L^{\infty}(\Omega) \neq \varnothing$. Let $f \in W \cap L^{\infty}(\Omega)$ and let $\Omega^{(f)}$ be the set of full measure consisting of all $\omega \in \Omega$ for which the sequence $\mathbf{a}=\left(f\left(T^{k} \omega\right)\right)$ has a correlation given by the spectral measure of $U_{T}$, via

$$
\lim _{n} \frac{1}{n} \sum_{j=0}^{n-1} f\left(T^{j+k} \omega\right) \overline{f\left(T^{j} \omega\right)}=\left\langle U_{T}^{k} f, f\right\rangle=\int_{\mathbf{C}_{1}} \cdot \lambda^{k} d\langle E(\lambda) f, f\rangle .
$$

This means that the spectral measure $\sigma_{\mathbf{a}}$ corresponding to $\mathbf{a}$ is continuous and thus, by Theorem 3.14 above, the bounded sequence $\mathbf{a}=\left(f\left(T^{k} \omega\right)\right)$ cannot be Besicovitch. Since this holds for each $\omega \in \Omega^{(f)}$, we have reached the desired contradiction and the proof is complete.

4. Sequences having a mean, as weights. To simplify matters we make precise some notation which will be used throughout the rest of the paper:

Notation. $d z$ will always denote Lebesgue measure on $\mathbf{C}_{1}$.

If $T \in C$, we denote by $U_{T}$ the associated unitary operator and by $E(\cdot)$ the corresponding spectral measure; for $f \in L^{2}(\Omega)$ we shall often write $\mu_{f}(\cdot)=\langle E(\cdot) f, f\rangle$.

We begin with the following elementary result.

LEMMA 4.1. Let $\mathbf{a}=\left(a_{n}\right)$ be a bounded complex sequence. Then:

$$
\begin{gathered}
\int_{\mathbf{C}_{1}}\left|\sum_{j=0}^{n-1} a_{j} z^{j}\right|^{2} d z=O(n) ; \\
\sum_{n=1}^{\infty} \int_{\mathbf{C}_{1}}\left|\frac{1}{n^{2}} \sum_{j=0}^{n^{2}-1} a_{j} z^{j}\right|^{2} d z<+\infty .
\end{gathered}
$$

In particular

$$
\sum_{n=1}^{\infty}\left|\frac{1}{n^{2}} \sum_{j=0}^{n^{2}-1} a_{j} z^{j}\right|^{2}<+\infty
$$

for almost every $z \in \mathbf{C}_{1}$ with respect to $d z$.

PROOF. We only need to remark that

$$
\int_{\mathbf{C}_{1}}\left|\sum_{j=0}^{n-1} a_{j} z^{j}\right|^{2} d z=\sum_{j=0}^{n-1}\left|a_{j}\right|^{2}=O(n)
$$

the rest follows immediately. 
THEOREM 4.2. Let $\mathbf{a}=\left(a_{n}\right)$ be any bounded complex sequence. Let $T \in \mathcal{C}$ and let $H_{1} \subset L^{2}(\Omega)$ correspond to the "absolutely continuous part' of the spectrum of $U_{T}$. Then for each $f \in H_{1}$ we have

$$
\frac{1}{n} \sum_{j=0}^{n-1} a_{j} f\left(T^{j} \omega\right) \rightarrow 0 \quad \text { a.e. }
$$

ProOF. Note that (see Lemma 2.9) $H_{1}$ is the closed vector space consisting of all $f \in L^{2}(\Omega)$ for which $\mu_{f} \ll d z$ and that

$$
G=\left\{f \in H_{1} \mid \mu_{f}=g d z \text { for some } 0 \leq g \leq 1\right\}
$$

generates a dense subspace of $H_{1}$. By Proposition 1.3 it suffices to consider $f \in G$. We have

$$
\begin{aligned}
\int_{\Omega}\left|\sum_{j=0}^{n-1} a_{j} f\left(T^{j} \omega\right)\right|^{2} d \mu(\omega) & =\sum_{(j, k)} a_{j} \bar{a}_{k} \int_{\Omega} f\left(T^{j} \omega\right) \overline{f\left(T^{k} \omega\right)} d \mu(\omega) \\
& =\sum_{(j, k)} a_{j} \bar{a}_{k} \int_{\mathbf{C}_{1}} z^{j} \bar{z}^{k} d \mu_{f}(z)=\int_{\mathbf{C}_{1}}\left|\sum_{j=0}^{n-1} a_{j} z^{j}\right|^{2} d \mu_{f}(z) \\
& =\int_{\mathbf{C}_{1}}\left|\sum_{j=0}^{n-1} a_{j} z^{j}\right|^{2} g(z) d z \leq \int_{\mathbf{C}_{1}}\left|\sum_{j=0}^{n-1} a_{j} z^{j}\right|^{2} d z .
\end{aligned}
$$

From statement (2) of Lemma 4.1 we deduce

$$
\sum_{n=1}^{\infty} \int_{\Omega}\left|\frac{1}{n^{2}} \sum_{j=0}^{n^{2}-1} a_{j} f\left(T^{j} \omega\right)\right|^{2} d \mu(\omega) \leq \sum_{n=1}^{\infty} \int_{\mathbf{C}_{1}}\left|\frac{1}{n^{2}} \sum_{j=0}^{n^{2}-1} a_{j} z^{j}\right|^{2} d z<+\infty
$$

and therefore

$$
\frac{1}{n^{2}} \sum_{j=0}^{n^{2}-1} a_{j} f\left(T^{j} \omega\right) \rightarrow 0 \quad \text { a.e. }
$$

The routine "subsequence argument" (see Proposition 1.9) now applies and completes the proof.

We recall that an automorphism $T \in C$ is said to have countable Lebesgue spectrum, or simply Lebesgue spectrum if there is a sequence $\left\{f_{j}\right\}_{j \in \mathbf{N}}$ of elements of $L^{2}(\Omega)$ such that $f_{0} \equiv 1$ and

$$
\left\{f_{0}\right\} \cup\left\{U_{T}^{n} f_{j} \mid j \geq 1, n \in \mathbf{Z}\right\}
$$

is an orthonornal basis of $L^{2}(\Omega)$. (It is known (see [38]) that every Kolmogorov automorphism has countable Lebesgue spectrum; in particular, every Bernoulli shift has countable Lebesgue spectrum.)

COROLlARY 4.3. Let $\mathbf{a}=\left(a_{n}\right)$ be a bounded complex sequence having a mean. Then $\mathbf{a}=\left(a_{n}\right)$ is a good weight (relative to the Weighted Pointwise Ergodic Theorem) for any $T \in C$ having Lebesgue spectrum.

PROOF. If $T \in C$ has Lebesgue spectrum, note that $H_{1}$ is precisely the orthogonal complement in $L^{2}(\Omega)$ of the constant functions and that, in turn, $L^{2}(\Omega)$ is dense in $L^{1}(\Omega)$ (use Proposition 1.3). 
REMARK. Compare Corollary 4.3 above with Theorem 2 in [14].

We now turn to a class of sequences introduced by J. Reich [34]. Let $J$ be a Borel set contained in $\mathbf{C}_{\mathbf{1}} \backslash\{1\}$.

DEFINITION 4.4. A bounded complex sequence $\mathbf{a}=\left(a_{n}\right)$ is said to satisfy a "uniform order condition" on $J$ if there is a sequence of positive integers $\left(N_{k}\right)$, dense in $\mathbf{N}$, such that

$$
D\left(J,\left(N_{k}\right)\right)=\sup _{z \in J}\left(\sum_{k=1}^{\infty}\left|\frac{1}{N_{k}} \sum_{j=0}^{N_{k}-1} a_{j} z^{j}\right|^{2}\right)<+\infty .
$$

The following result is essentially due to J. Reich [34].

PROPOSITION 4.5. Assume that the bounded complex sequence $\mathbf{a}=\left(a_{n}\right)$ satisfies a "uniform order condition" on J. Let $T \in C$. Then for each $f \in E(J) L^{2}(\Omega)$ we have

$$
\lim _{n} \frac{1}{n} \sum_{j=0}^{n-1} a_{j} f\left(T^{j} \omega\right)=0 \quad \text { a.e. }
$$

ProOF. We have $E\left(J^{c}\right) f=0$ and hence

$$
\begin{aligned}
\int_{\Omega}\left|\frac{1}{n} \sum_{j=0}^{n-1} a_{j} f\left(T^{j} \omega\right)\right|^{2} d \mu(\omega) & =\int_{\mathbf{C}_{1}}\left|\frac{1}{n} \sum_{j=0}^{n-1} a_{j} z^{j}\right|^{2} d \mu_{f}(z) \\
& =\int_{J}\left|\frac{1}{n} \sum_{j=0}^{n-1} a_{j} z^{j}\right|^{2} d \mu_{f}(z) .
\end{aligned}
$$

Thus

$$
\begin{aligned}
\sum_{k=1}^{\infty} \int_{\Omega}\left|\frac{1}{N_{k}} \sum_{j=0}^{N_{k}-1} a_{j} f\left(T^{j} \omega\right)\right|^{2} d \mu(\omega)= & \int_{J} \sum_{k=1}^{\infty}\left|\frac{1}{N_{k}} \sum_{j=0}^{N_{k}-1} a_{j} z^{j}\right|^{2} d \mu_{f}(z) \\
& \leq D\left(J,\left(N_{k}\right)\right) \mu_{k}(J)<+\infty
\end{aligned}
$$

It follows that

$$
\frac{1}{N_{k}} \sum_{j=0}^{N_{k}-1} a_{j} f\left(T^{j} \omega\right) \rightarrow 0 \text { a.e. }
$$

and the routine "subsequence argument" (see Proposition 1.9) completes the proof.

Again let $\mathbf{a}=\left(a_{n}\right)$ be a bounded complex sequence. Denote by $J(\mathbf{a})$ the collection of all Borel sets $J \subset \mathbf{C}_{1} \backslash\{1\}$ on which a satisfies a "uniform order condition". The following definition is a slight generalization of that given by Reich in [34].

DEFINITION 4.6. We say that a bounded complex sequence $\mathbf{a}=\left(a_{n}\right)$ "saturates" if there exists a sequence $\left(J_{n}(\mathbf{a})\right)$ of Borel sets in $J(\mathbf{a})$ such that

$$
K(\mathbf{a})=\mathbf{C}_{1} \backslash\left(\bigcup J_{n}(\mathbf{a})\right)
$$

is at most countable. 
REMARKS. (1) Let $\mathbf{a}=\left(a_{n}\right)$ be a bounded complex sequence and assume that there is a sequence of positive integers $\left(N_{k}\right)$, dense in $\mathbf{N}$, such that

$$
\sum_{k=1}^{\infty}\left|\frac{1}{N_{k}} \sum_{j=0}^{N_{k}-1} a_{j} z^{j}\right|^{2}<+\infty
$$

except for countably many $z \in \mathbf{C}_{1}$. Then $\mathbf{a}=\left(a_{n}\right)$ saturates.

(2) Let $\mathbf{a}=\left(a_{n}\right)$ be any bounded complex sequence. Then from Lemma 4.1 (and Proposition 1.9) we deduce that the amplitude exists,

$$
\Gamma(z)=\lim _{n} \frac{1}{n} \sum_{j=0}^{n-1} a_{j} z^{j}=0,
$$

for almost every $z \in \mathbf{C}_{1}$ with respect to $d z$.

(3) Assume that the bounded complex sequence $\mathbf{a}=\left(a_{n}\right)$ saturates. Then, as is easily seen, the amplitude exists,

$$
\Gamma(z)=\lim _{n} \frac{1}{n} \sum_{j=0}^{n-1} a_{j} z^{j}=0,
$$

for every $z \in \bigcup_{n} J_{n}(\mathbf{a})$, i.e. for all except countably many $z \in \mathbf{C}_{1}$.

The following is a slight generalization of a result in $[\mathbf{3 4}]$.

THEOREM 4.7. Assume that the bounded complex sequence $\mathbf{a}=\left(a_{n}\right)$ saturates. Let $T \in C$ and let $W=\left\{f \in L^{2}(\Omega) \mid \mu_{f}\right.$ is continuous $\}$. Then for each $f \in W$,

$$
\lim _{n} \frac{1}{n} \sum_{j=0}^{n-1} a_{j} f\left(T^{j} \omega\right)=0 \quad \text { a.e. }
$$

ProOF. By Proposition 1.3 it is enough to show that there exists a dense set in $W$ such that for each $g$ belonging to this set,

$$
\lim _{n} \frac{1}{n} \sum_{j=0}^{n-1} a_{j} g\left(T^{j} \omega\right)=0 \quad \text { a.e. }
$$

Let $f \in W$ and let $\varepsilon>0$. Let $\left(J_{n}(\mathbf{a})\right)$ be the sequence of Borel sets in $J(\mathbf{a})$ given by the saturation condition. Then $\mathbf{C}_{1} \backslash\left(\bigcup_{n} J_{n}(\mathbf{a})\right)$ is at most countable, and since $\mu_{f}$ is continuous,

$$
\mu_{f}\left(\mathbf{C}_{1} \backslash\left(\bigcup_{n} J_{n}(\mathbf{a})\right)\right)=0
$$

We may assume without loss of generality that the $J_{n}(\mathbf{a})$ 's are pairwise disjoint. Hence there exists $n$ such that

$$
\mu_{f}\left(\mathbf{C}_{1} \backslash\left(\bigcup_{l=0}^{n} J_{l}(\mathbf{a})\right)\right)<\varepsilon
$$


Now

$$
\begin{aligned}
\left\|f-\sum_{l=0}^{n} E\left(J_{l}(\mathbf{a})\right) f\right\|_{2}^{2} & =\left\|f-E\left(\bigcup_{l=0}^{n} J_{l}(\mathbf{a})\right) f\right\|_{2}^{2} \\
& =\left\langle E\left(\left(\bigcup_{l=0}^{n} J_{l}(\mathbf{a})\right)^{c}\right) f . f\right\rangle=\mu_{f}\left(\left(\bigcup_{l=0}^{n} J_{l}(\mathbf{a})\right)^{c}\right)<\varepsilon
\end{aligned}
$$

and it suffices to apply Proposition 4.5 to each one of the functions $g_{l}=E\left(J_{l}(\mathbf{a})\right) f$, $l=0, \ldots, n$.

Corollary 4.8. Let $\mathbf{a}=\left(a_{n}\right)$ be a bounded complex sequence. Assume that:

(i) a saturates.

(ii) The amplitude $\Gamma(z)=\lim _{n} \frac{1}{n} \sum_{j=0}^{n-1} a_{j} z^{j}$ exists for every $z \in \mathbf{C}_{1}{ }^{2}$

Then $\mathbf{a}$ is a good universal weight.

Proof. Let $T \in C$. By Proposition 1.3 it is enough to show that

$$
\lim _{n} \frac{1}{n} \sum_{j=0}^{n-1} a_{j} f\left(T^{j} \omega\right) \quad \text { exists a.e. }
$$

for $f$ belonging to a dense subset of $L^{2}(\Omega)$. By assumption (ii), (1) holds whenever $f$ is an eigenfunction of $U_{T}$. By Theorem 4.7, (1) holds for every $f$ belonging to $W=\left\{f \in L^{2}(\Omega) \mid \mu_{f}\right.$ is continuous $\}$. Since the vector space spanned by the eigenfunctions and by $W$ is dense in $L^{2}(\Omega)$, the proof is complete.

COROllary $4.9[\mathbf{1 1}]$. Let $\mathbf{a}=\left(a_{n}\right)$ be a bounded complex sequence. Assume that:

(i) a saturates.

(ii)

$$
\begin{aligned}
& \lim _{n} \frac{1}{n} \sum_{j=0}^{n-1} a_{j} z^{j}=0 \quad \text { for every } z \in \mathbf{C}_{1}, z \neq 1, \\
& \lim _{n} \frac{1}{n} \sum_{j=0}^{n-1} a_{j}=d .
\end{aligned}
$$

Then $\mathbf{a}$ is a good universal weight and

$$
\lim _{n} \frac{1}{n} \sum_{j=0}^{n-1} a_{j} f\left(T^{j} \omega\right)=d \int_{\Omega} f d \mu \quad \text { a.e. }
$$

for every $T \in C$ ergodic and $f \in L^{1}(\Omega)$.

PROOF. With the notation in the proof of Corollary 4.8 , it suffices to note that the equality $(*)$ holds for every eigenfunction and for every $f \in W$.

An example. Let $(X, X, P)$ be a probability space. For each integer $d \in \mathbf{N}$, $d \geq 1$, let $\mathcal{L}(d)=\{0, \pm d, \pm 2 d, \ldots\}$ be the lattice generated by $d$. A random variable $Y: X \rightarrow \mathbf{N}$ (or $Y: X \rightarrow \mathbf{Z}$ ) is said to be an $\mathcal{L}(d)$-lattice variable if

${ }^{2}$ By Remark (3) following Definition 4.6 this is then equivalent with requiring that the amplitude exists for every $z \in K(\mathbf{a})=\mathbf{C}_{1} \backslash\left(\bigcup_{n} J_{n}(\mathbf{a})\right)$. 
$P(\{Y \in \mathcal{L}(d)\})=1$ but there is no $d^{\prime}>d$ such that $P\left(\left\{Y \in \mathcal{L}\left(d^{\prime}\right)\right\}\right)=1$. It is well known and not hard to check that $Y$ is an $\mathcal{L}(1)$-lattice variable if and only if $E\left(e^{i \alpha Y}\right) \neq 1$ for all $0<\alpha<2 \pi$.

Now let $\left(Y_{n}\right)$ be a sequence of independent, indentically distributed random variables, $Y_{n}: X \rightarrow \mathbf{N}$. For each $k \in \mathbf{N}$ we write $S_{k}(x)=\sum_{l=0}^{k-1} Y_{l}(x)$ if $k \geq 1$ and $S_{0}(x) \equiv 0$. We now recall the following interesting result obtained by Blum and Cogburn [9].

Theorem 4.10 (Blum And CogBurn). Assume that $Y_{0}>0, E\left(Y_{0}\right)<+\infty$ and that $Y_{0}$ is an $\mathcal{L}(1)$-lattice variable (that is, $E\left(e^{i \alpha Y_{0}}\right) \neq 1$ for all $\left.0<\alpha<2 \pi\right)$. Then for each $0<\varepsilon<1$ there is a set $C_{\varepsilon} \in \mathcal{X}$ with $P\left(C_{\varepsilon}\right)=1$ such that for $x \in C_{\varepsilon}$

$$
\sup _{\substack{|z-1| \geq \varepsilon \\|z|=1}}\left|\frac{1}{n} \sum_{j=0}^{n-1} z^{S_{j}(x)}\right|=O\left(\frac{1}{n^{1 / 8}}\right) .
$$

For a proof see $[\mathbf{9}]$.

Under the assumption of Theorem 4.10 we also have, by the Strong Law of Large Numbers,

$$
\lim _{k} \frac{S_{k}(x)}{k}=E\left(Y_{0}\right)
$$

for $P$-almost every $x \in X$; thus the integer sequence $\left(S_{k}(x)\right)$ has density $d=$ $1 / E\left(Y_{0}\right)>0$ for $P$-almost every $x \in X$. It follows that there is a set $C \in X$ with $P(C)=1$ such that, for each $x \in C$, the weight associated with the integer sequence $\left(S_{k}(x)\right)$ (as in Proposition 1.7) satisfies the assumptions of Corollary 4.9 and hence is a good universal weight; this result is due to Blum and Reich (see [11]).

REMARKS. (1) By analogy with the terminology used by Blum and Eisenberg for subsequences of positive integers (see for instance $[\mathbf{1 1}]$ ), we call a bounded complex sequence $\mathbf{a}=\left(a_{n}\right)$ ergodic if

$$
\begin{aligned}
& \lim _{n} \frac{1}{n} \sum_{j=0}^{n-1} a_{j} z^{j}=0 \quad \text { for every } z \in \mathbf{C}_{1}, z \neq 0, \\
& \lim _{n} \frac{1}{n} \sum_{j=0}^{n-1} a_{j}=1
\end{aligned}
$$

Note that if the weight $\mathbf{a}=\left(a_{n}\right)$ is ergodic, then the Mean Ergodic Theorem holds for $\mathbf{a}=\left(a_{n}\right)$ : the limit and equality in relation $(*)$ (Corollary 4.9) holds in the $L^{1}$-mean with $d=1$ for every $T \in C$ ergodic and $f \in L^{1}(\Omega)$.

(2) Carrying over to weights the terminology used by Blum and Reich in [11] for subsequences of positive integers, we call a bounded complex sequence $\mathbf{a}=$ $\left(a_{n}\right)$ strictly ergodic if it is ergodic and it is a good universal weight. With this terminology, Corollary 4.9 is a slight generalization of the main theorem in [11]; it says that if a bounded weight $\mathbf{a}=\left(a_{n}\right)$ is ergodic and saturates, then it is strictly ergodic. 
5. Sequences having a correlation, as weights. We begin with the following result.

THEOREM 5.1. Let $\mathbf{a}=\left(a_{n}\right)$ be a bounded complex sequences having a mean and a correlation, and let $\lambda_{\mathbf{a}}$ be its spectral measure. Consider the following assertions:

(i) There is a sequence $\left(B_{p}\right)$ of Borel subsets of $\mathbf{C}_{1} \backslash\{1\}$ such that

$$
\lambda_{\mathbf{a}}\left(\bigcup_{p} B_{p}\right)=\lambda_{\mathbf{a}}\left(\mathbf{C}_{1} \backslash\{1\}\right)
$$

and for each fixed $p$,

$$
\int_{B_{p}}\left|\sum_{j=0}^{n-1} a_{j} \bar{z}^{j}\right|^{2} d \lambda_{\mathbf{a}}(z)=O(n) .
$$

(ii) There is a sequence of positive integers $\left(N_{k}\right)$, dense in $\mathbf{N}$, such that

$$
\sum_{k=1}^{\infty}\left|\frac{1}{N_{k}} \sum_{j=0}^{N_{k}-1} a_{j} \bar{z}^{j}\right|^{2}<+\infty \text { for } \lambda_{\mathbf{a}} \text {-a.e. } z \in \mathbf{C}_{1} \backslash\{1\} \text {. }
$$

(iii) The sequence $\mathbf{a}$ is a good universal weight. Then we have (i) $\Rightarrow$ (ii) $\Rightarrow$ (iii).

ProOF. (i) $\Rightarrow$ (ii). It suffices to take $N_{k}=k^{2}$ and to note that for each fixed $p$,

$$
\sum_{k=1}^{\infty} \int_{B_{p}}\left|\frac{1}{N_{k}} \sum_{j=0}^{N_{k}-1} a_{j} \bar{z}^{j}\right|^{2} d \lambda_{a}(z)<+\infty
$$

(ii) $\Rightarrow$ (iii). By Theorem 1.4 it suffices to consider $T \in C$ ergodic.

Let $H$ be the orthogonal complement of the constants in $L^{2}(\Omega)$. Since $\mathbf{a}=\left(a_{n}\right)$ has a mean, it suffices to show that for each $f \in H$,

$$
\lim _{n} \frac{1}{n} \sum_{j=0}^{n-1} a_{j} \overline{f\left(T^{j} \omega\right)}=0 \text { a.e. }
$$

By Lemma 2.9 we have the orthogonal decomposition of $H, H=H_{1} \oplus H_{2}$, where

$$
H_{1}=\left\{f \in H \mid \mu_{f} \prec \lambda_{\mathbf{a}}\right\}, \quad H_{2}=\left\{f \in H \mid \mu_{f} \perp \cdot \lambda_{\mathbf{a}}\right\} .
$$

Consider first the case of $H_{1}$. By (3), for each $f \in H_{1}$,

$$
\mu_{f}\left(\left\{\left.z \in \mathbf{C}_{1} \backslash\{1\}\left|\sum_{k=1}^{\infty}\right| \frac{1}{N_{k}} \sum_{j=0}^{N_{k}-1} a_{j} \bar{z}^{j}\right|^{2}=+\infty\right\}\right)=0
$$

thus if we set

$$
B_{p}=\left\{\left.z \in \mathbf{C}_{1} \backslash\{1\}\left|\sum_{k=1}^{\infty}\right| \frac{1}{N_{k}} \sum_{j=0}^{N_{k}-1} a_{j} \bar{z}^{j}\right|^{2} \leq p\right\}
$$


and take $p$ large enough, $\mu_{f}\left(B_{p}^{c}\right)$ can be made as small as we wish. Hence we may assume that $f \in H_{1}$ is such that $f=E(B) f$ with $B$ Borel contained in $B_{p}$ and $\mu_{f}=g d \lambda_{a}$ with $0 \leq g \leq p$ (these functions are dense in $H_{1}$ ). We have

$$
\begin{aligned}
\int_{\Omega}\left|\frac{1}{n} \sum_{j=0}^{n-1} a_{j} \overline{f\left(T^{j} \omega\right)}\right|^{2} d \mu(\omega) & \\
& =\int_{\mathbf{C}_{1}}\left|\frac{1}{n} \sum_{j=0}^{n-1} a_{j} \bar{z}^{j}\right|^{2} d \mu_{f}(z)=\int_{B}\left|\frac{1}{n} \sum_{j=0}^{n-1} a_{j} \bar{z}^{j}\right|^{2} d \mu_{f}(z) \\
& =\int_{B}\left|\frac{1}{n} \sum_{j=0}^{n-1} a_{j} \bar{z}^{j}\right|^{2} g(z) d \lambda_{\mathbf{a}}(z) \leq p \int_{B}\left|\frac{1}{n} \sum_{j=0}^{n-1} a_{j} \bar{z}^{j}\right|^{2} d \lambda_{\mathbf{a}}(z) .
\end{aligned}
$$

This implies

$$
\begin{aligned}
& \sum_{k=1}^{\infty} \int_{\Omega}\left|\frac{1}{N_{k}} \sum_{j=0}^{N_{k}-1} a_{j} \overline{f\left(T^{j} \omega\right)}\right|^{2} d \mu(\omega) \\
& \leq p \int_{B} \sum_{k=1}^{\infty}\left|\frac{1}{N_{k}} \sum_{j=0}^{N_{k}-1} a_{j} \bar{z}^{j}\right|^{2} d \lambda_{\mathbf{a}}(z) \leq p^{2} \lambda_{\mathbf{a}}(B)<+\infty
\end{aligned}
$$

and hence

$$
\frac{1}{N_{k}} \sum_{j=0}^{N_{k}-1} a_{j} \overline{f\left(T^{j} \omega\right)} \rightarrow 0 \quad \text { a.e. }
$$

The routine "subsequence argument" (Proposition 1.9) then yields

$$
\frac{1}{n} \sum_{j=0}^{n-1} a_{j} \overline{f\left(T^{j} \omega\right)} \rightarrow 0 \quad \text { a.e. }
$$

Assume now that $f \in H_{2}$. By the Individual Ergodic Theorem and the spectral theorem for $U_{T}$,

$$
\lim _{n} \frac{1}{n} \sum_{j=0}^{n-1} f\left(T^{j+k} \omega\right) \bar{f}\left(T^{j} \omega\right)=\int_{\mathbf{C}_{1}} z^{k} d \mu_{f}(z)
$$

for almost all $\omega \in \Omega$. This means that for any such $\omega$ the sequence $\left(b_{k}\right)$ defined by $b_{k}=f\left(T^{k} \omega\right)$ has a correlation and the corresponding spectral measure is $\mu_{f}$. Since $\lambda_{\mathbf{a}}$ and $\mu_{f}$ are mutually singular, it follows from Corollary 2.4 that

$$
\lim _{n} \frac{1}{n} \sum_{j=0}^{n-1} a_{j} \overline{f\left(T^{j} \omega\right)}=0 \text { a.e. }
$$

This finishes the proof. 
As a corollary of Theorem 5.1 we deduce

THEOREM 5.2. Let $\mathbf{a}=\left(a_{n}\right)$ be a bounded complex sequence having a mean and a correlation, and let $\lambda_{\mathbf{a}}$ be its spectral measure. If $\lambda_{\mathbf{a}}$ is absolutely continuous with respect to Lebesgue measure, then a is a good universal weight.

ProOF. We show that assertion (i) of Theorem 5.1 holds. We have $\lambda_{\mathbf{a}}=g d z$. Let $B_{p}=\left\{z \in \mathbf{C}_{1} \backslash\{1\} \mid g(z) \leq p\right\}$ for each $p \in \mathbf{N}$. For fixed $p$ we have

$$
\begin{aligned}
\int_{B_{p}}\left|\sum_{j=0}^{n-1} a_{j} \bar{z}^{j}\right|^{2} d \lambda_{\mathbf{a}}(z) & =\int_{B_{p}}\left|\sum_{j=0}^{n-1} a_{j} \bar{z}^{j}\right|^{2} g(z) d z \leq p \int_{B_{p}}\left|\sum_{j=0}^{n-1} a_{j} \bar{z}^{j}\right|^{2} d z \\
& \leq p \int_{\mathbf{C}_{1}}\left|\sum_{j=0}^{n-1} a_{j} \bar{z}^{j}\right|^{2} d z=p\left(\sum_{j=0}^{n-1}\left|a_{j}\right|^{2}\right)=O(n) .
\end{aligned}
$$

We note that (see also Lemma 2.9) if $T \in \mathcal{C}$ has countable Lebesgue spectrum, then for each $f \in L^{2}(\Omega)$ with $\int f d \mu=0$, the corresponding spectral measure $\mu_{f}$ (recall that $\mu_{f}(\cdot)=\langle E(\cdot) f, f\rangle$ ) is absolutely continuous with respect to Lebesgue measure: $\mu_{f} \ll d z$.

Theorem 5.2 then implies

Corollary 5.3. Let $T \in C$ have countable Lebesgue spectrum. Let $f \in L^{\infty}(\Omega)$ with $\int f d \mu=0$. Then there exists a set $\Omega_{f} \subset \Omega$ of probability one such that for every $\omega \in \Omega_{f}$, the sequence $\mathbf{u}=\left(u_{n}\right)$ given by $u_{n}=f\left(T^{n} \omega\right)$ has mean zero, has a correlation, and its spectral measure $\lambda_{\mathbf{u}}=\mu_{f}$; hence $\mathbf{u}$ is a good universal weight.

From Corollary 5.3 we immediately obtain

THEOREM 5.4. Let $T \in C$ have countable Lebesgue spectrum. Let $g \in L^{\infty}(\Omega)$. There exists then a set $\Omega_{g} \subset \Omega$ of probability one such that for every $\omega \in \Omega_{g}$ the sequence $\mathbf{v}=\left(v_{n}\right)$ given by $v_{n}=g\left(T^{n} \omega\right)$ is a good universal weight.

ProOF. Consider $f=g-\int g d \mu$.

REMARKS. (1) It is known that the class of automorphisms with countable Lebesgue spectrum is strictly larger than that of Kolmogorov automorphisms (see $[38$, p. 109, and 32]). There are, in fact, automorphisms with countable Lebesgue spectrum and zero entropy; such automorphisms cannot be Kolmogorov. For specific examples see [32].

(2) The special case of Theorem 5.4, when $T \in C$ is a Kolmogorov automorphism, was obtained, using different methods, by H. Furstenberg, M. Keane, J. P. Thouvenot and B. Weiss (personal communication).

6. Sequences of positive integers of density zero and the Individual Ergodic Theorem. We now return to the problem of the Individual Ergodic Theorem along subsequences. We shall be concerned here only with sequences of positive integers having density zero (for sequences of positive integers having nonzero density we know that the Individual Ergodic Theorem may be reduced to a Weighted Pointwise Ergodic Theorem: Proposition 1.7).

For convenience we write $\mathbf{N}^{*}=\{1,2,3, \ldots\}$ and we consider only strictly increasing sequences of integers from $\mathbf{N}^{*}$. We shall use the notation $\mathbf{l}=\left(l_{k}\right), \mathbf{n}=$ $\left(n_{k}\right), \mathbf{m}=\left(m_{k}\right)$ for such sequences. 
We need some more preliminaries. Let $T \in \mathcal{C}, \mathbf{n}=\left(n_{k}\right)$ and $p \in \mathbf{N}^{*}$. We set

$$
T_{p, \mathbf{n}} f(\omega)=\frac{1}{p} \sum_{k=1}^{p} f\left(T^{n_{k}} \omega\right) \quad \text { for } f \in L^{1}(\Omega) .
$$

For each $f \in L^{1}(\Omega)$ and $\lambda>0$ we set

$$
\begin{aligned}
& M_{\infty}(\mathbf{n}, T, f)(\omega)=\sup _{p \in \mathbf{N}^{*}}\left|T_{p, \mathbf{n}} f(\omega)\right|, \\
& E_{\infty}(\mathbf{n}, T, f, \lambda)=\left\{\omega \mid M_{\infty}(\mathbf{n}, T, f)(\omega)>\lambda\right\},
\end{aligned}
$$

and for $q \in \mathbf{N}^{*}$

$$
\begin{aligned}
& M_{q, \infty}(\mathbf{n}, T, f)(\omega)=\sup _{\substack{p \in \mathbf{N}^{*} \\
p \geq q}}\left|T_{p, \mathbf{n}} f(\omega)\right| \\
& E_{q, \infty}(\mathbf{n}, T, f, \lambda)=\left\{\omega \mid M_{q, \infty}(\mathbf{n}, T, f)(\omega)>\lambda\right\} .
\end{aligned}
$$

We recall J. P. Conze's important result on the Weak Maximal Inequality.

THEOREM $6.1[\mathbf{1 4}]$. With each sequence $\mathbf{n}=\left(n_{k}\right)$ one can associate a minimal constant $0<C(\mathbf{n}) \leq+\infty$ satisfying the inequality

$$
\lambda \mu\left(E_{\infty}(\mathbf{n}, T, f, \lambda) \leq C(\mathbf{n})\|f\|_{1}\right.
$$

for all $T \in C, f \in L^{1}(\Omega), \lambda>0$. Furthermore, $C(\mathbf{n})<+\infty$ if and only if there exists $T \in \mathcal{C}$ ergodic (resp. aperiodic) such that

$$
\lim _{p} T_{p, \mathbf{n}} f(\omega) \text { exist a.e. }
$$

for each $f \in L^{1}(\Omega)$ (that is, the Individual Ergodic Theorem holds for $T$ along the subsequence $\mathbf{n})$.

We recall now (see $[\mathbf{2 9}, \mathbf{1 4}]$ ):

DEFINITION 6.2. A sequence $\mathbf{n}=\left(n_{k}\right)$ is called "bad universal" (relative to the Individual Ergodic Theorem) if $C(\mathbf{n})=+\infty$; equivalently, if for each $T \in C$ ergodic (resp. aperiodic) there exists $f \in L^{1}(\Omega)$ such that $\left(T_{p, \mathbf{n}} f(\omega)\right)$ fails to converge a.e.

It is known that every lacunary sequence is "bad universal" (see [4]). Suppose now that we start with a lacunary sequence $\left(n_{k}\right)$ and that at each stage we add a block of consecutive integers

$$
n_{k}, n_{k}+1, \ldots, n_{k}+l_{k}
$$

How fast may the $l_{k}$ 's be allowed to grow, so that the resulting sequence is still "bad universal"? This is the question that we shall study next, using an elaboration of the methods in [4].

DEFINITION 6.3. Let $\mathbf{n}=\left(n_{k}\right)$ and $\mathbf{l}=\left(l_{k}\right)$ be strictly increasing sequences of integers from $\mathbf{N}^{*}$ such that $l_{k}<n_{k+1}-n_{k}$ for all $k \in \mathbf{N}^{*}$. Then the "block sequence" $\mathbf{m}$ generated by $\mathbf{n}$ and $\mathbf{l}$ is defined to be the sequence

$$
n_{1}, n_{1}+1, \ldots, n_{1}+l_{1}, \ldots, n_{k}, n_{k}+1, \ldots, n_{k}+l_{k}, \ldots
$$

We refer to $C_{k}=\left\{n_{k}, n_{k}+1, \ldots, n_{k}+l_{k}\right\}$ as the $k$ th block of $\mathbf{m}$.

We now come to the main results of this section. To simplify matters we divide the proof into several lemmas. 
In what follows, $\mathbf{m}$ will usually denote the "block sequence" generated by $\mathbf{n}=\left(n_{k}\right)$ and $\mathbf{l}=\left(l_{k}\right)$.

Let $p \in \mathbf{N}^{*}$ be given. Then starting with any $k_{0} \in \mathbf{N}^{*}, k_{0}>1$, define

$$
B_{-1}=\left\{n_{k}, n_{k}+1, \ldots, n_{k}+l_{k} \mid 1 \leq k<k_{0}\right\}
$$

and then define the following successive "composite blocks" $\left(k_{0}<k_{1}<k_{2}<\cdots<\right.$ $\left.k_{p}\right)$ :

$$
\begin{aligned}
& B_{0}=\left\{n_{k}, n_{k}+1, \ldots, n_{k}+l_{k} \mid k_{0} \leq k<k_{1}\right\}, \\
& B_{1}=\left\{n_{k}, n_{k}+1, \ldots, n_{k}+l_{k} \mid k_{1} \leq k<k_{2}\right\}, \\
& \vdots \\
& B_{p-1}=\left\{n_{k}, n_{k}+1, \ldots, n_{k}+l_{k} \mid k_{p-1} \leq k<k_{p}\right\}
\end{aligned}
$$

by induction, as follows:

We define $k_{1}$ to be the minimal integer $>k_{0}$ for which $\left|B_{0}\right|<\left|B_{-1}\right|$. Assuming $k_{1}<k_{2}<\cdots<k_{j}$ defined (hence $B_{0}, B_{1}, \ldots, B_{j-1}$ defined) we define $k_{j+1}$ to be the minimal integer $>k_{j}$ such that $\left|B_{j}\right|>\left|B_{-1}\right|+\left|B_{0}\right|+\cdots+\left|B_{j-1}\right|$.

With the above notation we have

LEMMA 6.4. Assume that the sequence $\left(l_{k}\right)$ satisfies the condition

$$
l_{k \prime}+1 \leq \sum_{k<k \prime}\left(l_{k}+1\right) \quad \text { for all } k^{\prime} \geq k_{0}
$$

Let $p \in \mathbf{N}^{*}$. Then we have

$$
\left|B_{-1}\right|+\left|B_{0}\right|+\cdots+\left|B_{j}\right| \leq 4^{j+1}\left|B_{-1}\right| \text { for } 0 \leq j \leq p-1 .
$$

In particular

$$
l_{k}<4^{p}\left|B_{-1}\right|=4^{p}\left(\sum_{h<k_{0}}\left(l_{h}+1\right)\right) \quad \text { for all } k<k_{p} .
$$

PROOF. Using condition $(\alpha)$ and the minimal choice of $k_{j+1}$ we have, for $0 \leq$ $j \leq p-1$,

$$
\begin{aligned}
\left|B_{j}\right| & =\sum_{k_{j} \leq k<k_{j+1}}\left(l_{k}+1\right)=\left(l_{k_{j+1}-1}+1\right)+\sum_{k_{j} \leq k<k_{j+1}-1}\left(l_{k}+1\right) \\
& \leq \sum_{k<k_{j+1}-1}\left(l_{k}+1\right)+\sum_{k_{j} \leq k<k_{j+1}-1}\left(l_{k}+1\right) \\
& =\sum_{k<k_{j}}\left(l_{k}+1\right)+2\left(\sum_{k_{j} \leq k<k_{j+1}-1}\left(l_{k}-1\right)\right) \\
& \leq\left(\left|B_{-1}\right|+\left|B_{0}\right|+\cdots+\left|B_{j-1}\right|\right)+2\left(\left|B_{-1}\right|+\left|B_{0}\right|+\cdots+\left|B_{j-1}\right|\right) \\
& =3\left(\left|B_{-1}\right|+\left|B_{0}\right|+\cdots+\left|B_{j-1}\right|\right) .
\end{aligned}
$$

We thus showed that for $0 \leq j \leq p-1$

$$
\left|B_{j}\right| \leq 3\left(\left|B_{-1}\right|+\left|B_{0}\right|+\cdots+\left|B_{j-1}\right|\right) \text {. }
$$

Now (1) follows from (3) by induction. In fact, for $j=0$ we have trivially

$$
\left|B_{-1}\right|+\left|B_{0}\right| \leq\left|B_{-1}\right|+3\left|B_{-1}\right|=4\left|B_{-1}\right| \text {. }
$$


Assuming now that inequality (1) holds for $j$, we derive it for $j+1$ :

$$
\begin{aligned}
\left|B_{-1}\right|+\left|B_{0}\right|+\cdots+\left|B_{j}\right|+\left|B_{j-1}\right| & \leq 4^{j+1}\left|B_{-1}\right|+3\left(\left|B_{-1}\right|+\left|B_{0}\right|+\cdots+\left|B_{j}\right|\right) \\
& \leq 4^{j+1}\left|B_{-1}\right|+3 \cdot 4^{j+1}\left|B_{-1}\right|=4^{j+2}\left|B_{-1}\right| .
\end{aligned}
$$

Thus (1) is proved. As (2) is an immediate consequence of (1), the lemma is proved.

LEMMA 6.5. Assume that the sequence $\left(l_{k}\right)$ satisfies

$$
l_{k_{0}}+1>\sum_{k<k_{0}}\left(l_{k}+1\right)
$$

Let $p \in \mathbf{N}^{*}$. Then we have

$$
\begin{gathered}
k_{1}-k_{0}=1 \\
k_{j+1}-k_{j} \leq 2\left(k_{j}-k_{j-1}\right) \quad \text { for } 1 \leq j \leq p-1 .
\end{gathered}
$$

In particular,

$$
k_{p}<k_{0}+2^{p}
$$

Proof. (4) follows immediately from $(\boldsymbol{x})$ and the minimal choice of $k_{1}$.

(5) By the monotone increasing property of the $l_{k}$ 's we have, for $1 \leq j \leq p-1$,

$$
\begin{aligned}
\sum_{k_{j} \leq k<k_{j}+2\left(k_{j}-k_{j-1}\right)}\left(l_{k}+1\right) & \geq 2\left(\sum_{k_{j-1} \leq k<k_{j}}\left(l_{k}+1\right)\right) \\
& =2\left|B_{j-1}\right|>\left|B_{j-1}\right|+\left|B_{-1}\right|+\left|B_{0}\right|+\cdots+\left|B_{j-2}\right| .
\end{aligned}
$$

From the minimal choice of $k_{j+1}$ we deduce $k_{j}+2\left(k_{j}-k_{j-1}\right) \geq k_{j+1}$, whence $k_{j+1}-k_{j} \leq 2\left(k_{j}-k_{j-1}\right)$.

(6) From (5) and (4) we get by induction

$$
k_{j+1}-k_{j} \leq 2\left(k_{j}-k_{j-1}\right) \leq \cdots \leq 2^{j}\left(k_{1}-k_{0}\right)=2^{j},
$$

and, summing over $j$,

$$
k_{p}-k_{1}=\sum_{j=1}^{p-1}\left(k_{j+1}-k_{j}\right) \leq \sum_{j=1}^{p-1} 2^{j}
$$

whence

$$
k_{p} \leq k_{1}+\sum_{j=1}^{p-1} 2^{j}=k_{0}+1+\sum_{j=1}^{p-1} 2^{j}<k_{0}+2^{p} .
$$

This finishes the proof.

LEMMA 6.6. Assume that the sequences $\left(n_{k}\right)$ and $\left(l_{k}\right)$ satisfy the conditions:

$$
\begin{gathered}
l_{k^{\prime}}+1 \leq \sum_{k<k^{\prime}}\left(l_{k}+1\right) \quad \text { for all } k^{\prime} \text { large enough, } \\
\sum_{k<k^{\prime}}\left(l_{k}+1\right)=o\left(n_{k^{\prime}}\right) .
\end{gathered}
$$


Let $p \in \mathbf{N}^{*}$ and $\delta>0$ be given. There is then $k_{0}$ large enough that ${ }^{3}$

$$
l_{k}<\delta p n_{k_{0}} \text { for all } k<k_{p} .
$$

Proof. By $(\alpha)$ and $(\beta)$, it suffices to choose $k_{0}$ large enough that $(\alpha)$ holds for all $k^{\prime} \geq k_{0}$ and

$$
\sum_{k<k_{0}}\left(l_{k}+1\right)<4^{-p} \delta p n_{k_{0}}
$$

and then to apply conclusion (2) of Lemma 6.4.

REMARK. With the notation of Lemma 6.6, under condition $(\alpha)$, condition $(\beta)$ is equivalent with the statement: The "block sequence" $\mathbf{m}$ has density zero (see also [5, Lemma 1.6]).

LemmA 6.7. Let $\Lambda \in R, \Lambda \geq 2$. Let $p+1=[\Lambda]\left(p \in \mathbf{N}^{*}\right)$. Assume that the sequences $\left(n_{k}\right)$ and $\left(l_{k}\right)$ satisfy the following conditions:

$$
l_{k \prime}+1>\sum_{k<k^{\prime}}\left(l_{k}+1\right) \quad \text { for infinitely many } k^{\prime} \in \mathbf{N}^{*} \text {. }
$$

Let $\delta>0$ be given. There is then $k_{0}$ large enough that

$$
l_{k}<\delta p n_{k_{0}} \quad \text { for all } k<k_{p} .
$$

Proof. By $\left(\gamma^{\prime}\right)$ there is a constant $c>0$ and $k_{0}$ large enough that $n_{k} / \Lambda^{k} \geq c$ for all $k \geq k_{0}$. By $\left(\beta^{\prime}\right)$ we may also choose $k_{0}$ large enough that

$$
l_{k} / \Lambda^{k} \leq \delta p \Lambda^{-2^{p}} c \text { for all } k \geq k_{0} .
$$

It follows that, for $k \geq k_{0}$,

$$
l_{k} \leq \delta \cdot p \cdot \Lambda^{k-2^{p}} c \leq \delta \cdot p \cdot \Lambda^{k-2^{p}}\left(n_{k_{0}} / \Lambda^{k_{0}}\right)=\delta \cdot p \cdot n_{k_{0}} \cdot \Lambda^{k-k_{0}-2^{p}} .
$$

By $\left(\boldsymbol{x}^{\prime}\right)$ we may also assume that

$$
l_{k_{0}}+1>\sum_{k<k_{0}}\left(l_{k}+1\right)
$$

Thus conclusion (6) of Lemma 6.5 applies, and (8) follows from (9) above, completing the proof.

Proposition 6.8. Let $A \in R, \Lambda \geq 2$. Let $p+1=[\Lambda]\left(p \in \mathbf{N}^{*}\right)$. Assume that the sequences $\left(n_{k}\right)$ and $\left(l_{k}\right)$ satisfy the following growth conditions:

$$
n_{k+1} / n_{k} \geq \Lambda \geq p+1 \text { for all } k \text { large enough. }
$$

$$
l_{k}=o\left(\Lambda^{k}\right)
$$

\footnotetext{
${ }^{3}$ With the previous notation.
} 
Then for the "block sequence" $\mathbf{m}$ generated by $\left(n_{k}\right)$ and $\left(l_{k}\right)$ the following holds: For each $q \in \mathbf{N}^{*}$ and each $\varepsilon>0$ there are $T \in \mathcal{C}$ and $A \in A$ with $\mu(A) \leq 2 / p$ such that

$$
\begin{aligned}
\mu\left(E_{q, \infty}\right. & \left.\left(\mathbf{m}, T, 1_{A}, \frac{1}{2}\right)\right) \\
& \geq \mu\left(\left\{\omega \mid \sum_{k=1}^{K} \sum_{t=0}^{l_{k}} 1_{A}\left(T^{n_{k}+t} \omega\right)>\frac{1}{2} \sum_{k=1}^{K}\left(l_{k}+1\right) \text { for some } K \geq 1\right\}\right) \\
& >1-\varepsilon .
\end{aligned}
$$

PROOF. By assumption $(*)$ we have $\Lambda^{k}=O\left(n_{k}\right)$ and using also $\left(\beta^{\prime}\right)$ we have $\left(\right.$ for $k^{\prime} \rightarrow \infty$ )

$$
\sum_{k<k \prime}\left(l_{k}+1\right)=o\left(\sum_{k<k^{\prime}} \Lambda^{k}\right)=o\left(\Lambda^{k \prime}\right)=o\left(n_{k \prime}\right) .
$$

Thus conditions $\left(\gamma^{\prime}\right)$ and $(\beta)$ of the previous lemmas are satisfied.

Let $q \in \mathbf{N}^{*}$ and $\varepsilon>0$ be given. Choose $\delta<\varepsilon / p$.

Now obviously the sequence $\left(l_{k}\right)$ must satisfy one of the (mutually exclusive) assumptions $(\alpha),\left(\boldsymbol{x}^{\prime}\right)$. Since all the other assumptions in Lemmas 6.6 and 6.7 are satisfied, it is clear that we can choose $k_{0}$ large enough that

$$
\begin{array}{ll}
k_{0} \geq q, & \\
n_{k+1} / n_{k} \geq p+1 & \text { for all } k \geq k_{0} \\
l_{k}<\delta p n_{k_{0}} & \text { for all } k<k_{p} .
\end{array}
$$

Now define for each $0 \leq j \leq p-1$ the set

$$
\Delta^{(j)}=\bigcup_{m \in \mathbf{Z}}\left[\frac{j}{p}+m, \frac{j+1}{p}+m\right]
$$

in $R$. If $n \in \mathbf{N}^{*}$ note that the set $\Delta_{n}^{(j)}=\left\{\omega \in \Omega \mid n \omega \in \Delta^{(j)}\right\}$ consists of intervals of the form $[j / p n+m / n,(j+1) / p n+m / n]$; these are intervals of length $1 / p n$ repeated periodically with period $1 / n$.

With the notation previously used, define now

$$
B_{j}^{\prime}=\left\{n_{k} \mid k_{j} \leq k<k_{j+1}\right\} \text { for } j=0, \ldots, p-1 .
$$

By the growth condition on $\left(n_{k}\right)$ (see $(10)$ ), it is easy to see that for any $0 \leq j^{\prime}$, $j^{\prime \prime} \leq p-1$ and $k \geq k_{0}$, every interval belonging to the set $\Delta_{n_{k}}^{(j \prime)}$ contains an interval belonging to the set $\Delta_{n_{k+1}}^{j \prime \prime}$. Hence we may choose

$$
a \in \bigcap_{j=0}^{p-1} \bigcap_{n \in B_{j}^{\prime}} \Delta_{n}^{(j)}, \quad a \in\left(0, \frac{1}{p n_{k_{0}}}\right)
$$

(that is, $a$ belonging to the interior of the foremost left interval of $\Delta_{n_{k_{0}}}^{(0)}$ ). From (10) and (11) we get

$$
l_{k} a<\delta \quad \text { for all } k<k_{p} .
$$

Below, for a real number $x$, we let $\{x\}$ denote the fractional part of $x$, that is, the residue of $x \bmod 1$. 
Define now $A=[0,2 / p], T \omega=\omega-a(\bmod 1)$. Then $T^{-m} A=A+m a(\bmod 1)$. For $m \in B_{0}$, we have $m=n_{s}+t$ with $k_{0} \leq s<k_{1}$ and $0 \leq t \leq l_{s}$. Since

$$
a \in \bigcap_{n \in B_{0}^{\prime}} \Delta_{n}^{(0)}=\Delta_{n_{k_{0}}}^{(0)} \cap \Delta_{n_{k_{0}+1}}^{(0)} \cap \cdots \cap \Delta_{n_{k_{1}-1}}^{(0)}
$$

we have $0 \leq\left\{n_{s} a\right\} \leq 1 / p$ and $t a<\delta$ (use (12)), so that

$$
0 \leq\left\{n_{s} a+t a\right\}<1 / p+\delta .
$$

As $T^{-m} A=A+n_{s} a+t a(\bmod 1)$, it follows that

$$
T^{-m} A \supset\left[\frac{1}{p}+\delta, \frac{2}{p}\right] \quad \text { for } m \in B_{0} ; \quad 1 / p \quad 2 / p
$$

Similarly for $m \in B_{j}(0<j \leq p-1)$ we have $m=n_{s}+t$ with $k_{j} \leq s<k_{j+1}$ and $0 \leq t \leq l_{s}$. Since

$$
a \in \bigcap_{n \in B_{j}^{\prime}} \Delta_{n}^{(j)}=\Delta_{n_{k_{j}}}^{(j)} \cap \Delta_{n_{k_{j}+1}}^{(j)} \cap \cdots \cap \Delta_{n_{k_{j+1}-1}}^{(j)}
$$

we have $j / p \leq\left\{n_{s} a\right\} \leq(j+1) / p$ and $t a<\delta$ (use (12)), so that

$$
j / p \leq\left\{n_{s} a+t a\right\}<(j+1) / p+\delta
$$

(for $j=p-1,\left\{n_{s} a+t a\right\} \in[1-1 / p, 1) \cup[0, \delta)$ ). As before, it follows that for each $0 \leq j \leq p-1$,

$$
m \in B_{j} \Rightarrow T^{-m} A \supset[(j+1) / p+\delta,(j+2) / p] \quad(\bmod 1) .
$$

From (13) and the definition of $k_{j}$, we obtain, for each $0 \leq j \leq p-1$,

$$
\begin{aligned}
\omega & \in\left[\frac{j+1}{p}+\delta, \frac{j+2}{p}\right](\bmod 1) \Rightarrow \frac{\sum_{1 \leq s<k_{j+1}} \sum_{0 \leq t \leq l_{s}} 1_{A}\left(T^{n_{s}+t} \omega\right)}{\sum_{1 \leq s<k_{j+1}}\left(l_{s}+1\right)} \\
& =\frac{1}{\left|B_{-1} \cup B_{0} \cup \cdots \cup B_{j}\right|}\left(\sum_{m \in B_{-1} \cup \cdots \cup B_{j}} 1_{A}\left(T^{m} \omega\right)\right) \\
& \geq \frac{1}{\left|B_{-1} \cup B_{0} \cup \cdots \cup B_{j}\right|}\left(\sum_{m \in B_{j}} 1_{A}\left(T^{m} \omega\right)\right) \geq \frac{\left|B_{j}\right|}{\left|B_{-1} \cup B_{0} \cup \cdots \cup B_{j}\right|}>\frac{1}{2} .
\end{aligned}
$$

'Thus

$$
\bigcup_{j=0}^{p-1}\left[\frac{j+1}{p}+\delta, \frac{j+2}{p}\right](\bmod 1) \subset E_{q, \infty}\left(\mathbf{m}, T, 1_{A}, \frac{1}{2}\right)
$$

and the proposition is proved.

THEOREM 6.9. Let $\mathbf{u}=\left(u_{j}\right)$ be a lacunary sequence of positive integers, that is, such that

$$
u_{j+1} / u_{j} \geq \lambda, \quad j \in \mathbf{N}^{*},
$$

for some constant $\lambda>1$. Let $1=\left(l_{j}\right)$ be an increasing sequence of positive integers such that $l_{j}<n_{j+1}-n_{j}$ for all $j \in \mathbf{N}^{*}$ and such that $\lim _{j} l_{j} / \lambda^{j}=0$. Then the "block sequence" $\mathbf{v}$ generated by $\mathbf{u}=\left(u_{j}\right)$ and $\mathbf{l}=\left(l_{j}\right)$, namely the sequence

$$
u_{1}, u_{1}+1, \ldots, u_{1}+l_{1}, u_{2}, u_{2}+1, \ldots, u_{2}+l_{2}, \ldots, u_{k}, u_{k}+1, \ldots, u_{k}+l_{k}, \ldots,
$$

is a bad universal sequence, that is, $C(\mathbf{v})=+\infty$. 
Proof. Let $r \in \mathbf{N}^{*}$ be large enough that $\lambda^{r} \geq 2$. Define

$$
\Lambda=\Lambda(r)=\lambda^{r}
$$

and

$$
p+1=[\Lambda] .
$$

Consider the sequence $\mathbf{n}=\left(n_{k}\right)_{k \in \mathbf{N}^{*}}$ defined by $n_{k}=u_{k r}$ and note that it satisfies the growth condition $(*)$ of Proposition 6.8 ,

$$
n_{k+1} / n_{k} \geq \Lambda \geq p+1 .
$$

Note also that the sequence $\left(l_{k r}\right)_{k \in \mathbf{N}^{*}}$ satisfies condition $\left(\beta^{\prime}\right)$ of Proposition 6.8:

$$
\lim _{k} \frac{l_{k r}}{\Lambda^{k}}=0 .
$$

Clearly also $l_{k r}<n_{k+1}-n_{k}$ for all $k \in \mathbf{N}^{*}$. Let $\mathbf{m}$ be the "block sequence" generated by $\left(n_{k}\right)$ and $\left(l_{k r}\right)_{k \in \mathbf{N}^{*}}$, namely

$$
n_{1}, n_{1}+1, \ldots, n_{1}+l_{r}, \ldots, n_{k}, n_{k}+1, \ldots, n_{k}+l_{k r}, \ldots
$$

Let $S \in \mathcal{C}, f \in L_{+}^{1}(\Omega)$ and compare the averages along $\mathbf{m}$ with the averages along v. We have

$$
\begin{aligned}
\sum_{k=1}^{K} \sum_{t=0}^{l_{k r}} f\left(S^{n_{k}+t} \omega\right) & =\sum_{k=1}^{K} \sum_{t=0}^{l_{k r}} f\left(S^{u_{k r}+t} \omega\right) \\
& \leq \sum_{k=1}^{K r} \sum_{t=0}^{l_{k}} f\left(S^{u_{k}+t} \omega\right)
\end{aligned}
$$

The number of terms $N(K)$ in $(\mathrm{I})$ is

$$
N(K)=\left(l_{r}+1\right)+\left(l_{2 r}+1\right)+\cdots+\left(l_{K r}+1\right) .
$$

The number of terms $N^{\prime}(K)$ in (II) satisfies

$$
N^{\prime}(K)<r\left(l_{r}+1\right)+r\left(l_{2 r}+1\right)+\cdots+r\left(l_{K r}+1\right) .
$$

Thus $N^{\prime}(K) / N(K)<r$. By Proposition 6.8 applied to $\mathbf{m}$, for each $\varepsilon>0$, there are $T \in \mathcal{C}$ and $A \in A$ with $\mu(A) \leq 2 / p$ such that

$$
\begin{aligned}
\mu\left(E_{\infty}\left(\mathbf{m}, T, 1_{A}, \frac{1}{2}\right)\right) \\
\quad \geq \mu\left(\left\{\omega \mid \frac{1}{N(K)} \sum_{k=1}^{K} \sum_{t=0}^{l_{k r}} f\left(T^{n_{k}+t} \omega\right)>\frac{1}{2} \text { for some } K \in \mathbf{N}^{*}\right\}\right) \\
>1-\varepsilon .
\end{aligned}
$$

By the estimate above

$$
\frac{1}{N(K)} \sum_{k=1}^{K} \sum_{t=0}^{l_{k r}} f\left(T^{n_{k}+t} \omega\right)>\frac{1}{2}
$$


implies

whence

$$
\frac{1}{N^{\prime}(K)} \sum_{k=1}^{K r} \sum_{t=0}^{l_{k}} f\left(T^{u_{k}+t} \omega\right)>\frac{1}{2 r}
$$

$$
\mu\left(E_{\infty}\left(\mathbf{v}, T, 1_{A}, \frac{1}{2 r}\right)\right)>1-\varepsilon
$$

By Theorem 6.1 we have, for all $a>0$,

$$
a \mu\left(E_{\infty}\left(\mathbf{v}, T, 1_{A}, a\right)\right) \leq C(\mathbf{v})\left\|1_{A}\right\|_{1} \leq C(\mathbf{v}) \cdot 2 / p .
$$

For $a=1 / 2 r$ we deduce $(1-\varepsilon) / 2 r \leq C(\mathbf{v}) \cdot 2 / p$, and, letting $\varepsilon \rightarrow 0, C(\mathbf{v}) \geq p / 4 r$. It follows from (14) and (15) that $p / r \rightarrow+\infty$, as we let $r \rightarrow+\infty$, and thus the theorem is proved.

REMARKS. (1) It was U. Krengel [29] who first introduced and proved the existene of "bad universal sequences". Subsequently various other mathematicians obtained the existence of "bad universal sequences" by different methods (see [14, 3, 4]). It follows from [14] (see Lemma 4, p. 11) that every bad universal sequence $\mathbf{n}=\left(n_{k}\right)$ must have lower density $0: l d(\mathbf{n})=0$.

(2) It was shown in [4] that every lacunary sequence is a bad universal sequence. Thus, in particular, the sequences $\left(2^{k}\right),(k !)$ are bad universal. But, in fact, more is true; each of the "block sequences" whose $k$ th block is given by

$$
\begin{array}{ll}
2^{k}, 2^{k}+1, \ldots, 2^{k}+k & (k \geq 1) \\
k !, k !+1, \ldots, k !+k & (k \geq 2)
\end{array}
$$

is bad universal (each of these sequences is: (a) "ergodic" in the sense of Blum and Reich [11]; (b) "saturates" in the sense of Reich [34]; and (c) has density zero). This answered a question raised by Blum and Reich [11] in their Concluding Remarks.

(3) It follows from Theorem 6.9 that there are bad universal "block sequences," whose blocks increase exponentially fast. In particular, for instance, the "block sequence" whose $k$ th block is given by

$$
3^{k}, 3^{k}+1,3^{k}+2, \ldots, 3^{k}+2^{k}
$$

is a bad universal sequence.

The above remarks might lead one to believe that every "block sequence" which has density zero is "bad universal". This is false, as was shown in [5]:

THEOREM 6.10. Assume that the "block sequence" $\mathbf{m}$ generated by $\mathbf{n}=\left(n_{k}\right)$ and $\mathbf{l}=\left(l_{k}\right)$, namely the sequence

$$
n_{1}, n_{1}+1, \ldots, n_{1}+l_{1}, \ldots, n_{k}, n_{k}+1, \ldots, n_{k}+l_{k}, \ldots
$$

satisfies the growth condition $l_{k} \geq C^{*} n_{k-1}($ for $k>1)$ for some constant $C^{*}>0$. Then $\mathbf{m}$ is a "good universal sequence" relative to the Individual Ergodic Theorem ${ }^{4}$ and the almost everywhere limit of the averages along this subsequence is precisely the projection operator onto the invariant functions.

For a proof see [5].

\footnotetext{
${ }^{4}$ We say, of course, that $\mathbf{m}=\left(m_{k}\right)$ is a "good universal sequence" relative to the Individual Ergodic Theorem if for each $T \in C \lim _{p} T_{p, \mathbf{m}} f(\omega)$ exists a.e., for all $f \in L^{1}(\Omega)$.
} 
REMARKS. (1) The "block sequence" $\mathbf{m}$ generated by $n_{k}=(k+1)$ ! and $l_{k}=k$ ! is a "good universal sequence" - of density zero-relative to the Individual Ergodic Theorem. This answers a question raised by H. Furstenberg: see [42, Problem 13 in Problem session] (see also [43, Chapter on Subsequence and Generalized Means, Pointwise Convergence Section, Good and Bad Sequences]).

(2) For further remarks and comments relating to the above questions see [5].

\section{BIBLIOGRAPHY}

1. H. Anzai and S. Kakutani, Bohr compactifications of a locally compact abelian group. I, II, Proc. Imp. Acad. (Tokyo) 19 (1943), 476-480, 533-539.

2. J. R. Baxter and J. H. Olsen, Weighted and subsequential ergodic theorems, Canad. J. Math. 35 (1983), 145-166.

3. A. Bellow, Sur la structure des suites "mauvaises universelles" en théorie ergodique, C. R. Acad. Sci. Paris Sér. I Math. 294 (1982), 55-58.

4. __ On "bad universal" sequences in ergodic theory (II), Proc. Univ. Sherbrooke Workshop on Measure Theory and Appl. (June 1982), Lecture Notes in Math., vol. 1033, Springer-Verlag, Berlin and New York, 1983.

5. A. Bellow and V. Losert, On sequences of density zero in ergodic theory, Proc. Conf. on Modern Analysis and Probability Honoring Prof. Shizuo Kakutani, Contemporary Math. Ser., vol. 26, Amer. Math. Soc., Providence, R.I., 1984, pp. 49-60.

6. J. P. Bertrandias, Suites pseudo-aléatoires et critères d'équirépartition module 1, Compositio Math. (Groningen) 16 (1964), 23-28.

7. __ Espaces des fonctions bornées et continues en moyenne asymptotique d'ordre p, Bull. Soc. Math. France 5 (1966), 1-106.

8. A. S. Besicovitch, Almost periodic functions, 2nd ed., Dover, New York, 1954.

9. J. R. Blum and R. Cogburn, on ergodic sequences of measures, Proc. Amer. Math. Soc. 51 (1975), 359-365.

10. J. R. Blum and J. I. Reich, The Individual Ergodic Theorem for p-sequences, Israel J. Math. 27 (1977), 180-184.

11. Strongly ergodic sequences of integers and the Individual Ergodic Theorem, Proc. Amer. Math. Soc. 86 (1982), 591-595.

12. H. Bohr, Almost periodic functions, Chelsea, New York, 1947.

13. A. Brunel and M. Keane, Ergodic theorems for operator sequences, Z. Wahrsch. Verw. Gebiete 12 (1969), 231-240.

14. J. P. Conze, Convergence des moyennes ergodiques pour des sous-suites, Bull. Soc. Math. France 35 (1973), 7-15.

15. J. Coquet, T. Kamae and M. Mendès France, Sur la mesure spectrale de certaines suites arithmétiques, Bull. Soc. Math. France 105 (1977), 369-384.

16. C. Corduneanu, Almost periodic functions, Wiley-Interscience, New York, 1968.

17. N. Dunford and J. T. Schwartz, Linear operators. I, Wiley, New York, 1958.

18. W. F. Eberlein, Abstract ergodic theorems and weak almost periodic functions, Trans. Amer. Math. Soc. 67 (1949), 217-240.

19. __ The point spectrum of weakly almost periodic functions, Michigan J. Math. 3 (1956), 137-139.

20 E. Følner, On the dual spaces of the Besiconitch almost periodic spaces, Mat.-Fys. Medd. Danske Vid. Selsk. 29 (1954).

21. M. Fréchet, Les fonctions asymptotiquement presque-périodiques continues, C. R. Acad. Sci. Paris Sér. I Math. 213 (1941), 520-522.

22. __ Les fonctions asymptotiquement presque-périodiques, Rev. Sci. 79 (1941), 341-354.

23. H. Furstenberg, Poincaré recurrence and number theory, Bull. Amer. Math. Soc. (N.S.) 5 (1981), 211234

24. I. Glicksberg and K. de Leeuw, Almost periodic functions on semigroups, Acta Math. 105 (1961), 99. 140.

25. C. C. Graham and O. C. McGehee, Essays in commutative harmonic analysis, Grundlehren Math. Wiss., Springer-Verlag, Berlin and New York, Vol. 238, 1979. 
26. M. de Guzmán, Real variable methods in Fourier analysis, North-Holland Math. Stud., Vol. 46, Amsterdam, New York, 1981.

27. P. R. Halmos and J. von Neumann, Operator methods in classical mechanics. II, Ann. of Math. (2) 43 (1942), 332-350.

28. A. Ionescu Tulcea and C. Ionescu Tulcea, Abstract ergodic theorems, Trans. Amer. Math. Soc. 107 (1963), 107-124.

29. U. Krengel, On the Individual Ergodic Theorem for subsequences, Ann. Math. Statist. 42 (1971), 1091-1095.

30. L. Kuipers and H. Niederreiter, Uniform distribution of sequences, Wiley-Interscience, New York, 1974.

31. V. Losert, On a class of sequences with a strong average property, Adv. in Math. (to appear).

32. D. Newton and W. Parry, On a factor automorphism of a normal dymamical system, Ann. Math. Statist. 37 (1966), 1528-1533.

33. J. C. Oxtoby, Ergodic sets, Bull. Amer. Math. Soc. 58 (1952), 116-136.

34. J. I. Reich, On the Individual Ergodic Theorem for subsequences, Ann. Probab. 5 (1977), 1039 1046.

35. V. A. Rohlin, On the fundamental ideas of measure theory, Amer. Math. Soc. Transl., Vol. 10, No. 1 (1962), 1-54.

36. C. Ryll-Nardzewski, Topics in ergodic theory, Lecture Notes in Math., Vol. 472, Springer-Verlag, 1975, pp. 131-156.

37. W. A. Veech, Commentary on $[\mathbf{4 1 a}, \mathbf{b}]$, N. Wiener: Collected Works, Vol. 1, Mathematicians of Our Time, M.I.T. Press, Cambridge, Mass., 1976.

38. P. Walters, An introduction to ergodic theory, Graduate Texts in Math. No. 79, Springer-Verlag, New York, 1982.

39. N. Wiener, Fourier integral and certain of its applications, Cambridge Univ. Press, London and New York, 1933.

40. N. Wiener and A. Wintner, Harmonic analysis and ergodic theory, Amer. J. Math. 63 (1941), 415-426.

41.

42. R. L. Adler, Ed., Ergodic theory and applications, Amer. Math. Soc. Summer Research Conf. (June 13-19, 1982, Univ. of New Hampshire, Durham), Amer. Math. Soc., Providence, R.I. (Informal Proceedings).

43. U. Krengel, Ergodic theorems, monograph, de Gruyter Stud. in Math., de Gruyter, Berlin (to appear). 60201

Department of MAThematics, NorthWEstern UNiversity, EVANSTON, Illinois

Institute of Mathematics, University of Vienna, A-1090 Vienna, Austria 